\title{
MyGIsFOS: an automated code for parameter determination and detailed abundance analysis in cool stars ${ }^{\star}$
}

\author{
L. Sbordone ${ }^{1,2}$, E. Caffau ${ }^{1,2, \star \star}$, P. Bonifacio ${ }^{2}$, and S. Duffau ${ }^{1}$ \\ 1 Zentrum für Astronomie der Universität Heidelberg, Landessternwarte, Königstuhl 12, 69117 Heidelberg, Germany \\ e-mail: 1sbordon@lsw.uni-heidelberg.de \\ 2 GEPI, Observatoire de Paris, CNRS, Université Paris Diderot, place Jules Janssen, 92190 Meudon, France
}

Received 2 August 2013 / Accepted 20 November 2013

\begin{abstract}
Context. The current and planned high-resolution, high-multiplexity stellar spectroscopic surveys, as well as the swelling amount of underutilized data present in public archives, have led to an increasing number of efforts to automate the crucial but slow process of retrieving stellar parameters and chemical abundances from spectra.

Aims. We present MyGIsFOS ${ }^{1}$, a code designed to derive atmospheric parameters and detailed stellar abundances from medium- to high-resolution spectra of cool (FGK) stars. We describe the general structure and workings of the code, present analyses of a number of well-studied stars representative of the parameter space MyGIsFOS is designed to cover, and give examples of the exploitation of MyGIsFOS very fast analysis to assess uncertainties through Monte Carlo tests.

Methods. MyGIsFOS aims to reproduce a "traditional" manual analysis by fitting spectral features for different elements against a precomputed grid of synthetic spectra. The lines of Fe I and Fe II can be employed to determine temperature, gravity, microturbulence, and metallicity by iteratively minimizing the dependence of Fe I abundance from line lower energy and equivalent width, and imposing Fe I-Fe II ionization equilibrium. Once parameters are retrieved, detailed chemical abundances are measured from lines of other elements.

Results. MyGIsFOS replicates closely the results obtained in similar analyses on a set of well-known stars. It is also quite fast, performing a full parameter determination and detailed abundance analysis in about two minutes per star on a mainstream desktop computer. Currently, its preferred field of application are high-resolution and/or large spectral coverage data (e.g., UVES, X-shooter, HARPS, Sophie).
\end{abstract}

Key words. methods: data analysis - techniques: spectroscopic - stars: fundamental parameters - stars: abundances

\section{Introduction}

The availability of several high-efficiency, high-multiplexity spectrographs has brought about the need to perform accurate abundance analysis on large sets of stellar spectra of low to high resolution. The problem has been tackled in many different ways, one may roughly divide the methods into global, which make use of the whole spectrum (e.g., Katz et al. 1998; Allende Prieto et al. 2000; Bailer-Jones 2000; Recio-Blanco et al. 2006) and local, that make use only of selected sections of the spectrum (e.g., Erspamer \& North 2002; Bonifacio \& Caffau 2003; Barklem et al. 2005; Boeche et al. 2011; Posbic et al. 2012; Mucciarelli et al. 2013; Magrini et al. 2013). A few intermediate cases exist, most notably SME (Spectroscopy Made Easy, Valenti \& Piskunov 1996; Barklem et al. 2005), underlining perhaps the difficulty of coming up with a clear-cut classification scheme. Complex pipelines, like that of the Sloan Digital Sky Survey (Allende Prieto et al. 2008), use multiple methods whose results are then suitably combined. Among the local codes one may distinguish between those that rely on equivalent widths (EWs) measured by automatic codes such as fitline (François et al. 2003), DAOSPEC (Stetson \& Pancino 2008) or ARES (Sousa et al. 2007) to determine stellar parameters and abundances (GALA, FAMA, Mucciarelli et al. 2013; Magrini et al. 2013), and those that rely on line fitting (Erspamer \& North 2002; Bonifacio \& Caffau 2003; Barklem et al. 2005; Boeche et al. 2011; Posbic et al. 2012; Van der Swaelmen et al. 2013).

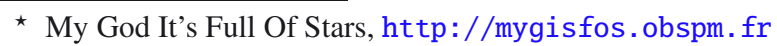
$\star \star$ Gliese Fellow.
}

Allende Prieto (2004) argued that EW based analysis should be abandoned; see also Bonifacio (2005) on EWs and line fitting.

We present in this paper the code MyGIsFOS that uses a local approach to treat large numbers of medium- to highresolution spectra. Among the local codes MyGIsFOS, Abbo (Bonifacio \& Caffau 2003), and the RAVE pipeline of Boeche et al. (2011) are the only ones, to our knowledge, that do not perform on-the-fly line transfer computations, but rely only on a precomputed grid. In the Boeche et al. (2011) pipeline, a library of synthetic curves of growth are compared to the measured EW of the chosen observed lines. In this sense, this code is closer to EW-based codes as far as line selection criteria, advantages, and limitations are concerned, but faster than most EW-based codes since no on-the-fly line transfer is performed.

On the other hand, MyGIsFOS and Abbo directly fit the synthetic profile of each chosen feature against the observed one. As such, they allow us to circumvent some of the limitations of EW-based codes (see, e.g., Sect. 5.1), while, at the same time, maintaining the speed advantage of codes not performing onthe-fly calculations.

At the same time, the RAVE pipeline, MyGIsFOS, and Abbo suffer limitations inherent to the use of pre-computed grids. Namely, these grids can become exceedingly large, or must be limited in parameter space range. Moreover, precomputed grids are by their nature rigid: their computation is resource intensive, and recomputation for the purpose of changing, for instance, the oscillation strengths of a couple of lines might not be a desirable option. 
For all these reasons, such codes are optimized to analyze a large number of stars that span a limited range in metallicity, effective temperature, and surface gravity.

MyGIsFOS specifically targets the treatment of large amounts of data, through a local approach, based on spectrum synthesis, line profile fitting, and a precomputed synthetic spectrum grid. Although other procedures exist that incorpoarate these features, none exists that uses all of these features at the same time. In this case we believe MyGIsFOS is unique and innovative.

\section{The purpose of MyGIsFOS}

MyGIsFOS is built on the foundation of our previous automatic abundance analysis code Abbo (Bonifacio \& Caffau 2003). Although we have completely rewritten the code with a different input-output system, and it is now considerably more powerful and faster, the scope of the code is unchanged with respect to Abbo. Broadly speaking, we compare the observed spectrum against a suitable grid of synthetic stellar spectra which we computed at varying $T_{\text {eff }}, \log g, V_{\text {turb }},[\mathrm{Fe} / \mathrm{H}]$, and $[\alpha / \mathrm{Fe}]$. Selected Fe I, Fe II, and $\alpha$-elements features are used to iteratively estimate the best values for each parameter, after which features for other elements are fitted to derive the corresponding abundances.

MyGIsFOS is conceived to strictly replicate a classical or manual procedure to derive stellar atmospheric parameters and detailed chemical abundances from high-resolution stellar spectra of cool stars. As such, its most typical usage can be summarized as follows:

- For each star to be analyzed, the user provides input observed spectrum and a set of first guess parameters.

- The user provides the code a feature list, i.e., a list of spectral intervals for the grid to be fitted against the observed spectrum. In the most general case, the feature list will include continuum intervals for pseudonormalization and signal-tonoise ratio $(\mathrm{S} / \mathrm{N})$ estimation, intervals corresponding to $\mathrm{Fe} \mathrm{I}$, Fe II, and $\alpha$-element lines for atmospheric parameter and metallicity $([\mathrm{Fe} / \mathrm{H}])$ determination, and intervals corresponding to lines of other elements for the determination of detailed abundances.

- $T_{\text {eff }}$ is determined from a set of isolated Fe I lines imposing the linear fit of the transition lower energy vs. line abundance to have null slope (for brevity, we will henceforth refer to this quantity as lower energy abundance slope, or LEAS).

- $V_{\text {turb }}$ is determined by imposing null slope for the EW vs. abundance relation of isolated Fe I lines.

- $\log g$ is determined from Fe I-Fe II ionization equilibrium.

- Fe abundance is determined from Fe I lines only.

- $[\alpha / \mathrm{Fe}]$ is determined by measuring lines of various $\alpha$ elements, and using their average $[\mathrm{X} / \mathrm{Fe}]$ as an estimate of $[\alpha / \mathrm{Fe}]$.

- $V_{\text {turb }}, \log g$, and $[\alpha / \mathrm{Fe}]$ are estimated iteratively, in a nested fashion, $[\alpha / \mathrm{Fe}]$ being the outermost "shell", $V_{\text {turb }}$ the innermost. For a given set of current guesses of $T_{\text {eff }}, \log g$, and $\alpha$ enhancement, $V_{\text {turb }}$ is determined, then the code proceeds to update the gravity estimate: if the current one is not appropriate, a new one is guessed, $V_{\text {turb }}$ is redetermined by assuming the new gravity and the existing estimates for the other parameters, then gravity is tested again. When a satisfactory estimate is reached for both $V_{\text {turb }}$ and $\log g,[\alpha / \mathrm{Fe}]$ is tested, and if changed, a recalculation of $V_{\text {turb }}$ and $\log g$ is triggered.
- $T_{\text {eff }}$ is evaluated in a slightly different fashion: initially, the aforementioned analysis is repeated to full convergence assuming a set of different temperatures, and in each case, the LEAS is derived. This intial mapping is used to fit the $T_{\text {eff }}{ }^{-}$ LEAS relationship and to look for a zero-slope temperature, which is then used to repeat the $V_{\text {turb }}, \log g$, $[\alpha / \mathrm{Fe}]$ determination. The LEAS is evaluated again and the estimated slope is added to the $T_{\text {eff }}$-LEAS relationship fitting sample. A zero is searched again and so on, until convergence is reached.

- Any of the aforementioned parameters can be either derived as described from the spectrum, or kept fixed at a userdefined value. MyGIsFOS will, of course, refrain from altering parameters for which the corresponding estimator is absent (e.g., gravity will not be estimated if no Fe II features are successfully measured). Thus, the user does not need to provide features for any parameter he/she is not planning to alter, the only exception being Fe I features, which should always be present ([Fe/H] cannot be kept fixed).

- The whole analysis is executed in a fully automated way for all the stars in the input list and the output is stored in a separate directory for each star. The input star list should contain data of similar quality and spectral range (e.g., UVES redarm spectra with $S / N \sim 50-100$; for a description of UVES, see Dekker et al. 2000), and of objects of comparable characteristics (say, metal-rich FGK dwarfs) because feature list, general running parameters (e.g., fit rejection tolerances) and synthetic spectra grid are common to the input star list and should thus be appropriate for all the objects. The constraints on $\mathrm{S} / \mathrm{N}$, spectral type and metallicity, are not very tight. Experiments with real UVES spectra have shown that if the feature list has been optimized for $\mathrm{S} / \mathrm{N}$ in the range $50-100$ it can work well for $\mathrm{S} / \mathrm{N}$ in the ratio $15-300$. At a low $\mathrm{S} / \mathrm{N}$ most of the selected features are not detected because they are too weak and the user has to switch to a selection of strong, saturated lines. At the high $\mathrm{S} / \mathrm{N}$ end it is useful to add many weak lines that are not detectable at lower $\mathrm{S} / \mathrm{N}$. For atmospheric parameters, the main constraint is metallicity, since features that are heavily blended become much cleaner and are usable at lower metallicity; at the same time, features that are saturated in the high-metallicity regime reach the linear part of the curve of growth at lower metallicity. The switchover between a metal-rich and metal-weak regime happens somewhere between $[\mathrm{M} / \mathrm{H}]-0.5$ and -1.0 . MyGIsFOS is designed to analyze data sets for which we have some previous knowledge (colors, metallicity estimates, membership to a cluster or dwarf galaxy). Our experience is that in these cases the stars that need to be rerun a second time because they are initially misclassified are less than $5 \%$ of the total.

It is then clear that the ideal application of MyGIsFOS is the determination of detailed chemical abundances from high- to intermediate-resolution spectra of cool stars with basically the same limitations and strengths as a traditional manual analysis: the results will be of higher quality if the spectral coverage is large, if $\mathrm{S} / \mathrm{N}$ is good, and if clean, unblended features are chosen. Few, noisy, or unreliable Fe II lines will make gravity estimation difficult, the quality of the adopted atomic data impacts the precision of any abundance derived, and so on. On the other hand, MyGIsFOS results are immediately comparable with the results derived from a traditional abundance analysis. Uncertainties, limitations, and dependence on the assumption made in atmosphere modeling and spectrosynthesis are well known, and researchers in the field are long since used to dealing with them. This makes MyGIsFOS output easy to test, interpret, 
Table 1. The MyGIsFOS grids computed with SYNTHE and ATLAS 12 models employed for the present paper.

\begin{tabular}{|c|c|c|c|c|c|c|}
\hline $\begin{array}{l}\text { Grid } \\
\text { name }\end{array}$ & $\begin{array}{l}T_{\text {eff }} \\
\text { range } \\
{[\mathrm{K}]} \\
\end{array}$ & $\begin{array}{l}\log g \\
\text { range } \\
\text { [c.g.s.] }\end{array}$ & $\begin{array}{l}V_{\text {turb }} \\
\text { range } \\
{\left[\mathrm{km} \mathrm{s}^{-1}\right]}\end{array}$ & $\begin{array}{l}{[\mathrm{Fe} / \mathrm{H}]} \\
\text { range }\end{array}$ & $\begin{array}{l}{[\alpha / \mathrm{Fe}]} \\
\text { range }\end{array}$ & $\begin{array}{l}\text { Number of } \\
\text { models }\end{array}$ \\
\hline $\begin{array}{l}\text { Metal poor cool dwarfs } \\
\text { (MPCD) }\end{array}$ & $\begin{array}{l}5000 \text { to } 6000 \\
\text { step } 200 \mathrm{~K}\end{array}$ & $\begin{array}{l}3 \text { to } 5 \\
\text { step } 0.5\end{array}$ & $\begin{array}{l}0 \text { to } 3 \\
\text { step } 1\end{array}$ & $\begin{array}{l}-4 \text { to }-0.5 \\
\text { step } 0.5\end{array}$ & $\begin{array}{l}-0.4 \text { to } 0.8 \\
\text { step } 0.4\end{array}$ & 3840 \\
\hline $\begin{array}{l}\text { Metal rich cool dwarfs } \\
\text { (MRCD) }\end{array}$ & $\begin{array}{l}5000 \text { to } 6000 \\
\text { step } 200 \mathrm{~K}\end{array}$ & $\begin{array}{l}3 \text { to } 5 \\
\text { step } 0.5\end{array}$ & $\begin{array}{l}0 \text { to } 3 \\
\text { step } 1\end{array}$ & $\begin{array}{l}-1 \text { to } 0.75 \\
\text { step } 0.25\end{array}$ & $\begin{array}{l}-0.4 \text { to } 0.8 \\
\text { step } 0.4\end{array}$ & 3840 \\
\hline $\begin{array}{l}\text { Metal poor giants } \\
\text { (MPG) }\end{array}$ & $\begin{array}{l}4200 \text { to } 5600 \\
\text { step } 200 \mathrm{~K}\end{array}$ & $\begin{array}{l}1 \text { to } 3 \\
\text { step } 0.5\end{array}$ & $\begin{array}{l}1 \text { to } 3 \\
\text { step } 1\end{array}$ & $\begin{array}{l}-4 \text { to }-0.5 \\
\text { step } 0.5\end{array}$ & $\begin{array}{l}-0.4 \text { to } 0.4 \\
\text { step } 0.4\end{array}$ & 2880 \\
\hline $\begin{array}{l}\text { Metal rich giants } \\
\text { (MRG) }\end{array}$ & $\begin{array}{l}4200 \text { to } 5600 \\
\text { step } 200 \mathrm{~K}\end{array}$ & $\begin{array}{l}1 \text { to } 3 \\
\text { step } 0.5\end{array}$ & $\begin{array}{l}1 \text { to } 3 \\
\text { step } 1\end{array}$ & $\begin{array}{l}-1 \text { to } 0.5 \\
\text { step } 0.25\end{array}$ & $\begin{array}{l}-0.4 \text { to } 0.4 \\
\text { step } 0.4\end{array}$ & 2520 \\
\hline
\end{tabular}

and compare with the results from previous works, which are advantages that MyGIsFOS shares with EW-based automation schemes such as FAMA or GALA.

MyGIsFOS produces an extensive output (in ASCII format) for each star to allow for critical examination of the analysis outcome. Included is detailed information on each feature fitted (best fitting line profile, abundance, EW, rejection flags and quality-of-fit estimators), pseudonormalized input spectra and best-fitting synthetic spectra, as well as averaged abundances and parameters in tabular form, and a full listing of all the input parameters, employed grid characteristics, and code version. Also, all output files from the same run share a timestamp to ease tracking.

Thanks to the use of a fully precomputed synthetic grid, MyGIsFOS is remarkably fast: a typical run on a standard desktop computer, with full parameter determination and 20 element abundances, for $200 \mathrm{~nm}$ high-resolution optical spectra takes about $120 \mathrm{~s}$ per star (see Sect. 8 for more details).

\section{The synthetic spectra grid}

MyGIsFOS works by comparing selected spectral features with a grid of synthetic stellar spectra with varying $T_{\text {eff }}, \log g, V_{\text {turb }}$, $[\mathrm{Fe} / \mathrm{H}]$, and $[\alpha / \mathrm{Fe}]$. The grid is provided as an unformatted binary file that reduces physical size and read-in time. The grid contains a header containing a comment, the grid metrics (starting point, step, and number of steps in each parameter), the assumed solar abundances, the elements affected by $\alpha$ enhancement and the assumed grid instrumental broadening. The grid should be fed to MyGIsFOS already broadened as needed by the spectrograph resolution. The grid is divided into several spectral ranges or frames. This is foreseen to handle situations in which only limited, noncontiguous spectral ranges are needed, such as when treating data from different settings of single-order, highmultiplexity spectrographs such as VLT/FLAMES ${ }^{1}$ (Pasquini et al. 2002). If this is not needed, the grid might contain a single frame covering the needed spectral range. The frame subdivision is also indicated in the grid header, which contains all the information needed by MyGIsFOS to read in the grid data without user intervention. The grid should be passed to MyGIsFOS at high sampling $(\Delta \lambda / \lambda>100000)$ to prevent the introduction

\footnotetext{
1 Apart from the savings in file size, this allows the user to apply different instrumental broadening values to each frame as they are needed, e.g., for different VLT/FLAMES settings or VLT/X-shooter spectral arms.
}

of artifacts when it is resampled over the actual observed data points.

For all the tests presented in this paper, we computed a set of model atmospheres and synthetic spectra appropriate for the analysis of FGK stars, both dwarfs and giants, over a wide range of metallicities. The atmospheric models were computed with the Linux version of ATLAS 12, while synthetic spectra covering the UVES RED 580 setting (approx 480 to $680 \mathrm{~nm}$ ) were computed by means of SYNTHE (Kurucz 2005; Castelli 2005; Sbordone et al. 2004; Sbordone 2005). Parameter space coverage of the used grids to date are listed in Table 1. We computed models and syntheses in a self-consistent way, i.e. by including changes in $V_{\text {turb }}$ and $[\alpha / \mathrm{Fe}]$ already in the opacity computation during model calculation. We took solar abundances from Caffau et al. (2011b) for the elements there analyzed, and from Lodders et al. (2009) for the remaining species. The grids used are part of a larger set currently being prepared for publication (Sbordone et al., in prep.). Synthetic grids are computed with sampling $\Delta \lambda / \lambda=300000$ in the $460-690 \mathrm{~nm}$ range, and take in their binary packaged version between 1.5 and $2.2 \mathrm{~GB}$ of space.

\section{The MyGlsFOS workflow in detail}

As stated above, MyGIsFOS replicates classical manual abundance analysis workflow. User defined parameters are read from an ASCII parameter file. A flowchart of the process for a single star is shown in Fig.1, while an example of the result is given in Fig. 2. The workflow can be summarized as follows:

1. The observed spectrum (or spectra) is pseudonormalized by evaluating the pseudo-median of each continuum interval ${ }^{2}$. A continuum value is then calculated for each observed pixel by computing a spline through all the continuum intervals. Also, from every continuum interval the local $\mathrm{S} / \mathrm{N}$ is estimated. The whole synthetic grid is pseudonormalized in the same way. The pseudonormalization is kept fixed throughout the analysis of the star.

2 Given a vector of $N$ values, the median is defined as the value at the N/2-th element of the sorted vector. The pesudomedian employed by MyGIsFOS is instead the value at the $N / a$-th element of the sorted vector. It is thus equivalent to the true median for $a=2$, but the most typical values employed in MyGIsFOS are in the range $a=1.25-1.66$ : this is done to account for the fact that in most cases the chosen continuum intervals correspond more exactly to pseudocontinua, where weak lines are buried into the noise so that the use of a straight average or median would underestimate the continuum value. It is left to the user to fix the proper pseudomedian factor $a$ by visually inspecting the spectra. 


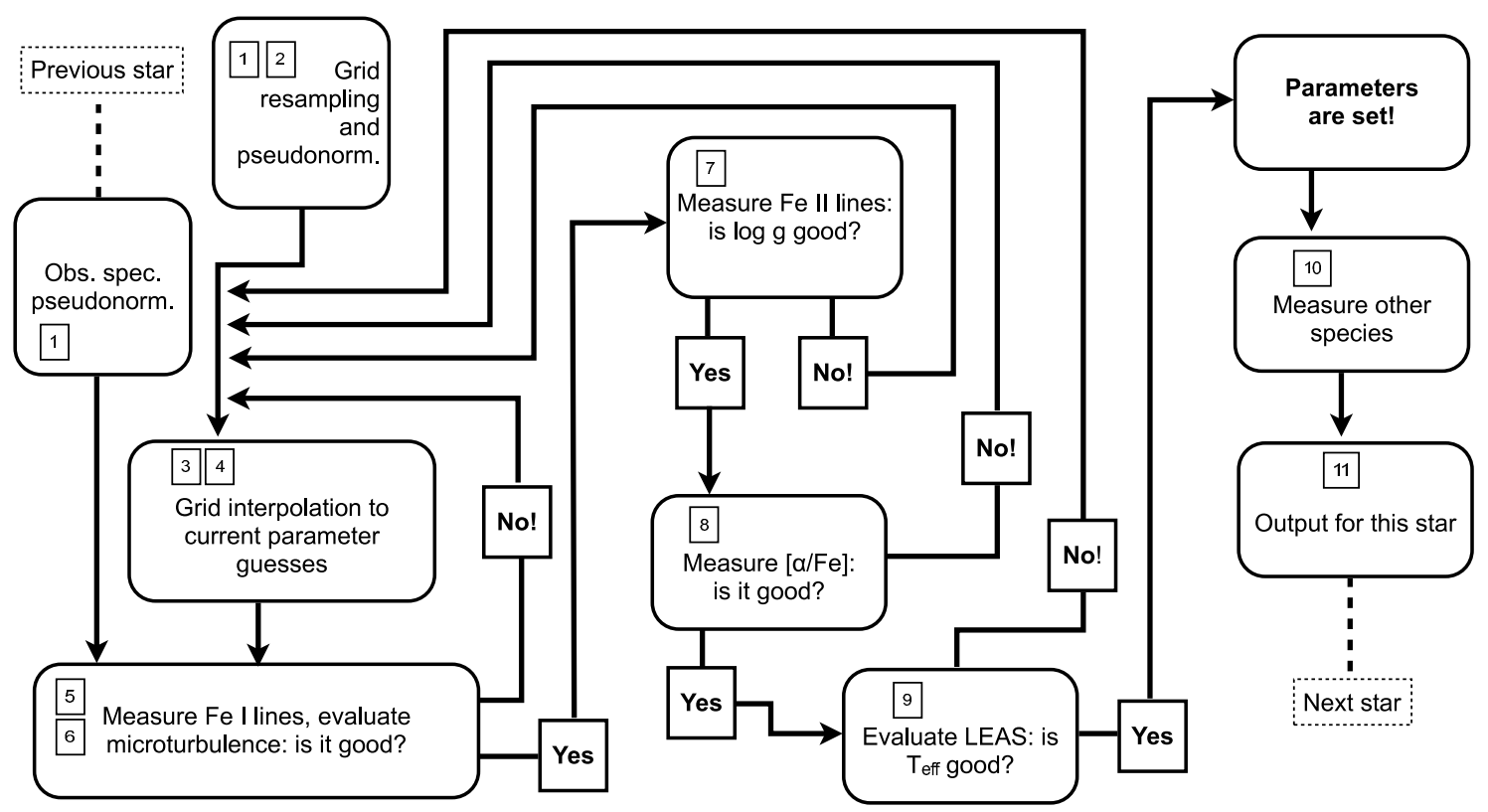

Fig. 1. A schematic flowchart of MyGIsFOS. Numbers in rectangles refer to the MyGIsFOS phases enumeration given in Sect. 4.

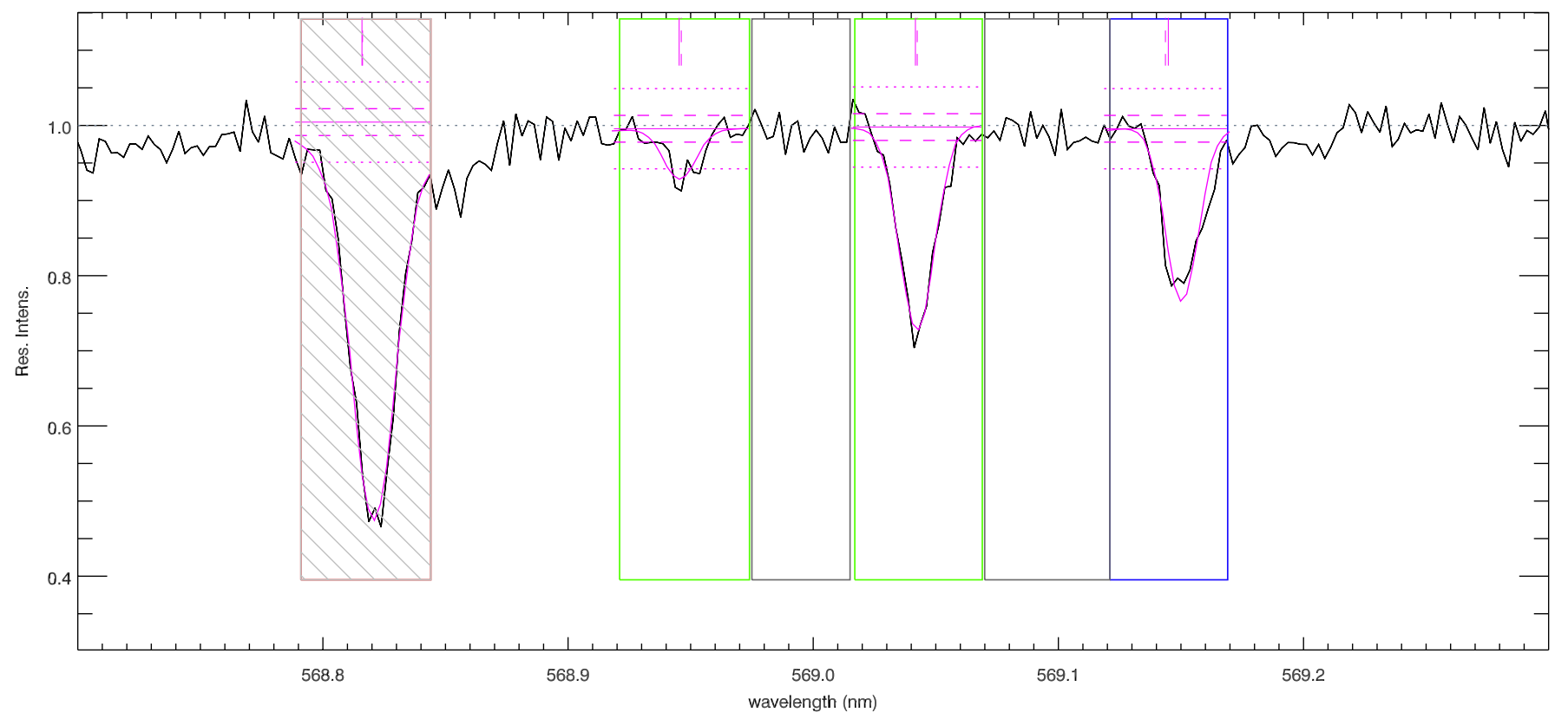

Fig. 2. A small section of the $S / N=50$ solar spectrum presented in 6.1 with overplotted MyGIsFOS features and fitting result as produced by the ancillary plotting package SQUID. From left to right: a rejected Na I line (rejected due to EW exceeding the applied maximum EW constraint, gray shaded box); a Ti I feature (green box); a continuum interval (gray box); a Si I feature (green box again); another continuum (gray); a Fe I features (blue box). The observed spectrum (black line) appears as renormalized by MyGIsFOS. The gray dashed horizontal line represents the continuum level, each feature is superimposed with the best fit synthetic (magenta), the best fit continuum (magenta continuous horizontal line), $1 \sigma$ and $3 \sigma$ values of the noise (horizontal dashed and dotted magenta lines), and markers of best fit doppler shift for the feature (magenta vertical continuous and dashed lines).

2. The synthetic grid is then resampled at the wavelengths of each observed pixel.

3. The first value for effective temperature estimation is chosen. If $T_{\text {eff }}$ is kept fixed for the star, said value is the initial guess provided as input, and is never changed afterward. Otherwise, MyGIsFOS begins scanning the $T_{\text {eff }}$ LEAS relationship. This can be performed in two modes: either each $T_{\text {eff }}$ in the grid is tried (full scan mode), or only a few hundred Ks around the initial estimate are tried (local scan mode). To do so, for each temperature probed $\log g, V_{\text {turb }}$,
$[\mathrm{Fe} / \mathrm{H}]$, and $[\alpha / \mathrm{Fe}]$ are determined (Steps between 4 and 8 below), then the LEAS is determined (Step 9 below). The operation is repeated for each $T_{\text {eff }}$ to be scanned. After the scan is completed, MyGIsFOS begins to refine its temperature estimate by fitting the $T_{\text {eff }}$-LEAS relationship with a 2 nd order polynomial and estimating the zero-LEAS $T_{\text {eff }}$ value. Each new attempt is added to the sample, the fit repeated, and the new zero determined. The search stops when stepto-step $T_{\text {eff }}$ change is less than a threshold parameter value (typically, $50 \mathrm{~K}$ ). 
4. At the beginning of the actual line measurement stage, the synthetic grid is interpolated at current guess values for $T_{\text {eff }}$, $\log g, V_{\text {turb }}$, and $[\alpha / \mathrm{Fe}]$. As a consequence, it is now reduced to a set of synthetic spectra with equal atmosphere parameters, but varying metallicity.

5. A set of Fe I lines is measured: for each feature, the set of synthetic spectra at varying metallicity is compared to the observed spectrum and the best fit determined by $\chi^{2}$ minimization allowing three free parameters: metallicity, a small ${ }^{3}$ radial velocity shift, and a small deviation from the established continuum value, never to surpass a given fraction of the local $\mathrm{S} / \mathrm{N}$. Local $\mathrm{S} / \mathrm{N}$ is evaluated by fitting a third order polynomial to all the $\mathrm{S} / \mathrm{N}$ estimates in the relevant observed frame. Also, EW is determined for both the observed and best fitting synthetic feature by direct integration under the pseudocontinuum. We use EWs in some of the line rejection criteria, and in the search for the best microturbulence, see below. Measurement for each feature is kept if a number of criteria are met: i) the fit probability should exceed a given threshold; ii) the EW of the observed and fitted synthetic line should not differ by more than a given threshold; iii) both the observed and the synthetic EWs should exceed a given value; iv) both EWs should exceed a certain number of times the EW of a noise-dominated line ${ }^{4}$; and v) EW should not exceed a user-defined maximum value $\mathrm{EW}_{\max }$, to avoid using heavily saturated lines. If any of the above tests fails, the feature is marked for rejection. The same rejection criteria will also be applied to features for every other element later on in the analysis.

6. Once all the assigned Fe I features are measured, the average of their abundance is computed, and $\sigma$-clipping performed (every feature deviating more than $n \sigma$ from the average is rejected, $n$ being set as one of MyGIsFOS input parameters). After the $\sigma$-clipping phase, a linear fit is performed in the $\mathrm{EW}-\mathrm{A}(\mathrm{Fe})$ plane to determine microturbulence. Steps 5 and 6 are performed at least four times: the first time measuring Fe I lines at the grid's lowest microturbulence value, the second time at the highest, the third at the zero of the linear fit of the first two, the fourth at the zero of the 2nd order fit of the first three. In a fashion similar to what is described for $T_{\text {eff }}$ in step 3 every time a new $V_{\text {turb }}$ is evaluated, it is added to the sample and a 2nd order polynomial is fitted again to the whole set. We do not seek the minimum slope of the linear $\mathrm{EW}-\mathrm{A}(\mathrm{Fe})$ relationship, but when it is smaller than the threshold set in the parameter file, microturbulence is considered determined, as well as Fe I abundance.

7. Step 4 is repeated again at the established microturbulence, and Fe II lines are measured. Their average, $\sigma$-clipped abundance is compared with the average Fe I abundance. If their discrepancy exceeds the threshold set in the parameter file, a new gravity is estimated from the size of said discrepancy and Steps 4-6 are repeated until a new value of microturbulence and $\mathrm{Fe} I$ abundance are found with the new gravity. The Fe I and Fe II abundances are compared again and the process is repeated until convergence is reached.

8. With the current estimates of microturbulence and gravity, lines are measured for all the $\alpha$ element ions chosen to

\footnotetext{
3 Currently, one quarter of synthetic grid broadening. E.g., $1.75 \mathrm{~km} \mathrm{~s}^{-1}$ for a grid broadened to $7 \mathrm{~km} \mathrm{~s}^{-1}$.

4 The EW of a noise-dominated line is computed as the EW of a triangular line whose depth corresponds to the local $1 \sigma \mathrm{S} / \mathrm{N}$, and whose FWHM is the same as either the grid instrumental broadening, or a userprovided value.
}

estimate $\alpha$ enhancement (we speak of ions because, for instance, one might choose to estimate $\alpha$ enhancement from $\mathrm{Mg}$ I and Ti II). Average, $\sigma$-clipped abundances for each ion are determined, and their respective $[\mathrm{X} / \mathrm{Fe}]$ are averaged to estimate $[\alpha / \mathrm{Fe}]$. Steps 4 to 7 are repeated and all the parameters are established again at the newly estimated $\alpha$ enhancement. The $\alpha$ enhancement itself is computed again and compared with the previous value. The process is iterated until the difference is below the threshold set in the parameter file.

9. The LEAS is determined with the current parameters, and MyGIsFOS goes back to Step 3 if $T_{\text {eff }}$ is being derived.

10. The full set of atmospheric parameters is established, and abundances are measured for all the features of all the other ions given in the linelists but not yet measured, again by $\chi^{2}$ fitting of the line profiles. When more than one featrure is available for a ion they are averaged, $\sigma$-clipped, and averaged again to determine the final value of the abundance of that ion.

11. Output files are created, and MyGIsFOS moves on to analyze the next star.

Summarizing the procedure is composed of several blocks that have to be iterated: Steps 3-9 can be viewed as the temperature block, Steps 5, 6 are the metallicity block, Step 6 is the microturbulence block, Steps 5-7 are the gravity block and Steps 5-8 are the alpha-enhancement block.

\subsection{Comments on the MyGIsFOS workflow}

The method for fixing the microturbulence is essentially the same as in Mucciarelli et al. (2013). The advantage of automatizing a "classical" approach, rather than a global $\chi^{2}$ minimization, such as in SME (Valenti \& Piskunov 1996) or TGMET (Katz et al. 1998) is that in the classical approach the different diagnostics (for $T_{\text {eff }}, \log g$, microturbulence, abundances) are kept separate, in a global $\chi^{2}$ minimization they are all considered together, and it is diffcult to break degeneracies. When information other than the spectra can be used (e.g., distances), some parameters can be easily and neatly fixed in the classical approach, less so in a global approach. MyGIsFOS has the same advantage over other, non $\chi^{2}$ based, global methods (Allende Prieto et al. 2000; Bailer-Jones 2000; Recio-Blanco et al. 2006) that are indeed very fast, but cannot easily break degeneracies. Van der Swaelmen et al. (2013) do not use $\chi^{2}$ fitting, but minimize another quantity, that is similar to $\chi^{2}$ (see their Sect. 4.1), as a consequence they cannot use the theorems of $\chi^{2}$ to estimate the goodness of fit.

\section{Specifics and limitations}

\subsection{Features vs. lines}

MyGIsFOS operates on spectral features rather than spectral lines. Technically, the fitter at its core compares an observed spectral range to a set of synthetic profiles of the same range varying in $[\mathrm{Fe} / \mathrm{H}]$ (more on this in Sect. 5.2), and determines the $[\mathrm{Fe} / \mathrm{H}]$ value for which the fit is the best. As such, MyGIsFOS can fit blended features without any problem, but, at the same time, it is unable to perform deblending.

The first characteristic comes in handy, e. g., when the user wants to derive an overall metallicity from low-resolution spectra because complex blends can be used as metallicity indicators. Another example is the case in which important lines (e. g., the only line available for an element one wants to measure) are 
blended with some other feature. And of course, MyGIsFOS has no problem fitting lines affected by hyper fine splitting (HFS), provided HFS was included when the synthetic grid was computed. Naturally, the quality of the fit of a blend depends on how well the atomic data of all the relevant features are known. It is also important to keep in mind that element-to-element ratios are kept fixed to the ones set in the grid (with the relevant exception of $[\alpha / \mathrm{Fe}])$, which could skew the result of fitting a blend if the two elements involved are not present in the star's atmosphere with a ratio similar to the one assumed in the grid.

The inability to perform deblending is relevant in any situation where the EW of a line is important, the most obvious case being $V_{\text {turb }}$ determination, which uses the EW of Fe I lines. MyGIsFOS determines two EWs for each feature it measures: one for the observed spectrum feature, and one for the bestfitting synthetic. Both are computed by direct integration under the local continuum value, and their difference is among the criteria used to reject a fit (see Sect. 4, point 5). For Fe I features, $\mathrm{EW}$ is then used to estimate $V_{\text {turb }}$. However, if a feature is a blend, its total EW will be too large in comparison to the associated abundance, skewing the $V_{\text {turb }}$ fit. For this reason, the user can decide which Fe I features to use for $V_{\text {turb }}$ estimation, and must exercise restraint to use only features corresponding to bona fide isolated Fe I lines.

In a similar fashion, the user indicates which $\mathrm{Fe} I$ lines to employ for $T_{\text {eff }}$ estimation. In a blend of two Fe I features, for instance, the user might not meaningfully associate one single lower energy value. Also, it is customary to refrain from using low excitation lines in $T_{\text {eff }}$ estimation, given their general tendency to be prone to stronger departures from local thermodynamical equilibrium (LTE). In diagnostic plots for the test stars (Sect. 6, Figs. 5 through 10), lines used or rejected in the $T_{\text {eff }}$ and $V_{\text {turb }}$ fitting are clearly indicated.

\subsection{Metallicity vs. abundance in line fitting}

MyGIsFOS computes abundances for lines of all elements by means of a grid that has only two degrees of freedom in chemical composition: metallicity and $\alpha$ enhancement. In fact, every line is fitted only against metallicity. Since all abundances scale the same way with metallicity in the grid (with the exception of $\alpha$ enhancement), MyGIsFOS interprets the result of the fit as due only to the change in the abundance of the specific ion producing the feature. By doing so, MyGIsFOS assumes that varying $[\mathrm{Fe} / \mathrm{H}]$, but keeping $[\mathrm{X} / \mathrm{Fe}]$ constant produces the same effect on the line profile than varying $[\mathrm{X} / \mathrm{Fe}]$ while keeping $[\mathrm{Fe} / \mathrm{H}]$ constant, or, in other words, it neglects the effect on the atmospheric structure of varying the overall star metallicity. This allows MyGIsFOS to drastically reduce the number of degrees of freedom in the grid, while still being able to measure abundances for an arbitrary number of ions. Otherwise, the grid should grow one dimension for every element that can be measured. This would either require that we use grids with a quite limited parameter span that is undesirable when searching for optimal atmospheric parameters, or that we use much larger grids, which are expensive to calculate, read in, and process inside MyGIsFOS. Moreover, with memory requirements being the bottleneck in running the code (see Sect. 8), such very large grids would rapidly become unwieldy.

The MyGIsFOS approximation thus remains valid as long as the measured abundance does not depart much from the grid solar-scaled composition, since this implies that the synthetic line profile is computed on the basis of an atmosphere that is not much different from the one providing the overall best parameter
Table 2. A test of the ability of MyGIsFOS to correctly retrieve $\mathrm{Ca}$ I abundances deviating from the assumed solar-scaled composition.

\begin{tabular}{ccrl}
\hline \hline $\begin{array}{c}\text { Input } \\
{[\mathrm{Ca} / \mathrm{Fe}]}\end{array}$ & $\begin{array}{c}\text { Offset from } \\
\text { model value }\end{array}$ & $\begin{array}{r}\text { Retrieved } \\
{[\mathrm{Ca} / \mathrm{Fe}]}\end{array}$ & $\sigma$ \\
\hline-0.5 & -0.9 & -0.52 & 0.05 \\
-0.2 & -0.6 & -0.23 & 0.05 \\
0.1 & -0.3 & 0.07 & 0.04 \\
0.4 & 0.0 & 0.39 & 0.04 \\
0.7 & 0.3 & 0.70 & 0.04 \\
1.0 & 0.6 & 1.00 & 0.04 \\
1.3 & 0.9 & 1.29 & 0.05 \\
\hline
\end{tabular}

fit. To provide an indicative estimate of how safe the approach is, we have computed a series of synthetic spectra around parameters typical of a metal poor dwarf $\left(T_{\mathrm{eff}}=5600 \mathrm{~K}, \log g=4.5\right.$, $\left.V_{\text {turb }}=1.0,[\mathrm{Fe} / \mathrm{H}]=-1.5,[\alpha / \mathrm{Fe}]=+0.4\right)$ and changing the $\mathrm{Ca}$ abundance to offsets of $\pm 0.3,0.6$, and 0.9 dex (since in the starting model $[\mathrm{Ca} / \mathrm{Fe}]=+0.4$, this corresponds to $[\mathrm{Ca} / \mathrm{Fe}]=-0.5$ to +1.3 , and $[\mathrm{Ca} / \mathrm{H}]=-2.0$ to -0.2 ). The spectra were noiseinjected to $S / N=80$ and fed to MyGIsFOS. First, the spectrum without $\mathrm{Ca}$ offset was analyzed with full parameter determination to determine how close MyGIsFOS would get to the input values, returning $T_{\text {eff }}=5580 \mathrm{~K}, \log g=4.41, V_{\text {turb }}=$ $0.88,[\mathrm{Fe} / \mathrm{H}]=-1.49,[\alpha / \mathrm{Fe}]=0.39$, values well in line with the typical offset to be expected as a result of noise injection (see 7.2). Then, the parameters were locked to the input values, and MyGIsFOS was used to derive abundances only, to see whether the Ca offsets would be retrieved properly despite the abundance-vs.-metallicity approximation. The result of the test are presented in Table 2 and, for a specific Ca I line, in Fig. 3. For the specific case, the approximation applied appears to produce no detectable effect on the ability of MyGIsFOS to properly measure abundances deviating for the assumed solar-scaled composition.

We want to stress that the presented case is not necessarily representative of any possible line being analyzed. For instance, if the line being fitted is significantly blended, in the synthetic grid being fitted against the observed both the main feature and the blending ones will vary, which would lead to skewed resulting abundances for any element deviating significantly from solar-scaled abundance. Also, the degree of sensitivity to the MyGIsFOS approximation depends on the physics of line formation for the specific feature. In Fig. 4, we show the comparison of three atmospheric models from our grid that are very close to the models of interest in this comparison. The central model is the one used to compute all the simulated observed spectra considered in this section $\left(T_{\text {eff }}=5600 \mathrm{~K}, \log g=4.5\right.$, $\left.V_{\text {turb }}=1.0,[\mathrm{Fe} / \mathrm{H}]=-1.5,[\alpha / \mathrm{Fe}]=+0.4\right)$. The other two differ in metallicity only, being offset by 1 dex upward and downward, thus being representative of the \pm 0.9 dex variation in metallicity of the models used in the fitting grid. In other terms, while all the synthetic profiles being fitted to our simulated observation, in the ideal case, should be drawn from the central model by only varying $\mathrm{Ca}$ abundance, they are instead drawn by models that differ in metallicity as much as the ones plotted in Fig. 4.

The CaI lines, such as the ones measured in this example, belong to a trace species in such atmospheres, and are thus temperature sensitive, but only weakly pressure sensitive. At the typical formation depths for weak $\operatorname{lines}\left(\log \left(\tau_{\text {ross }}\right)\right.$ roughly between -2 and 0$)$, the three models are quite close in temperature. On the other hand, the pressure is significantly different, but these lines are very weakly sensitive to it. Majority species lines (e.g., ionized element lines), or damped lines with stronger 
L. Sbordone et al.: A code for automatic abundance analysis

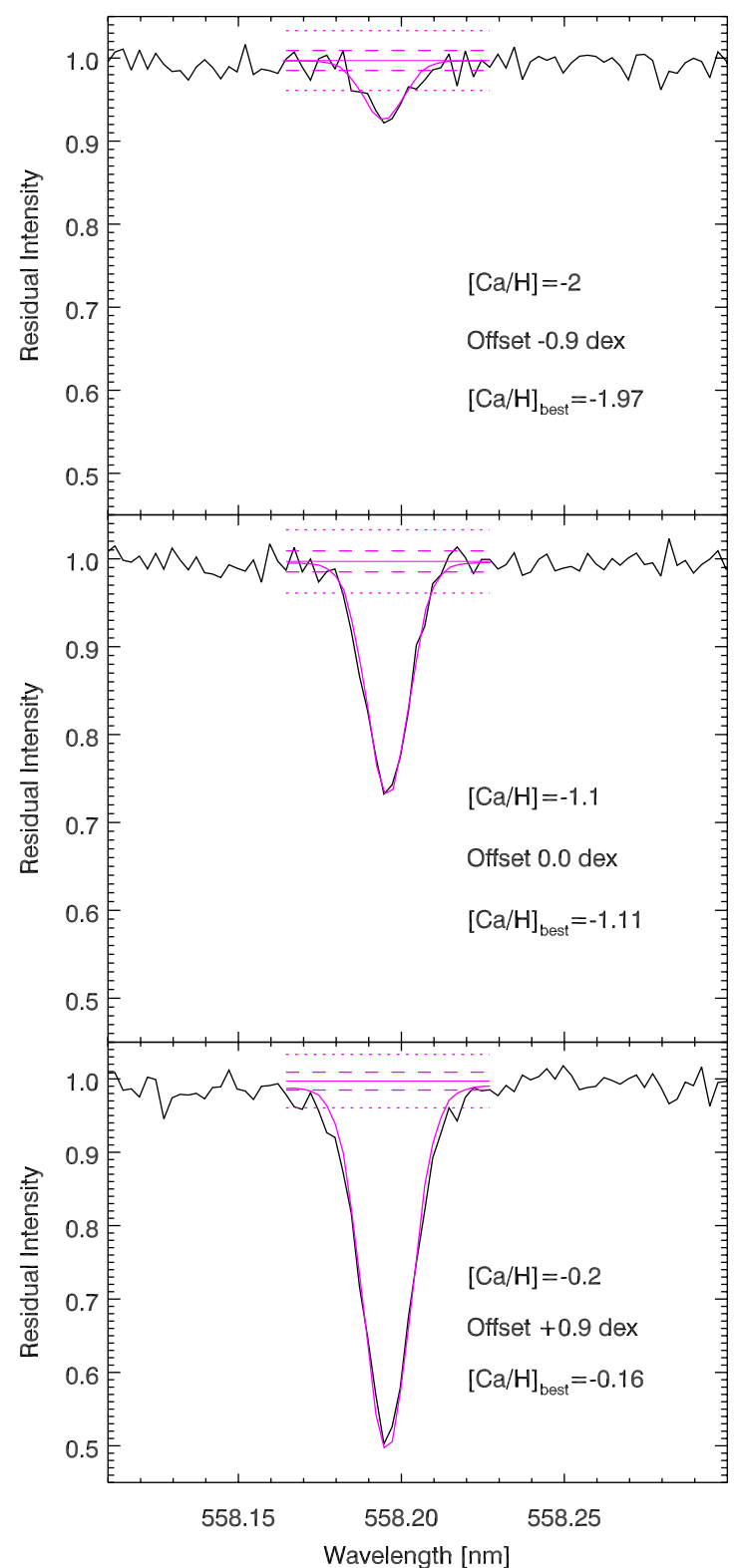

Fig. 3. The Ca I $559.19 \mathrm{~nm}$ line in the case of the two most extreme offsets described in Sect. 5.2 and in the no-offset case, with their respective best fitting synthetic value (magenta profiles). The magenta horizontal lines represent local continuum, one- $\sigma$, and three- $\sigma$ of the local simulated noise. $[\mathrm{Ca} / \mathrm{H}]$ is the input value, $[\mathrm{Ca} / \mathrm{H}]_{\text {best }}$ the best fit value for the line.

pressure sensitivity, are likely to display stronger departures in this situation. As such, we suggest that users verify the abundances derived by MyGIsFOS for species that depart heavily from grid solar-scaled values. However, since significant differences might arise only for species strongly departing from solar abundance ratios, the detection of abundance anomalies through MyGIsFOS is to be considered robust, and only the exact amount of the abundance departure might be in need of verification.

\section{Tests on reference stars}

As an assessment of MyGIsFOS performance, in this section we present parameter determination and chemical analysis for a few representative stars. To reproduce typical data characteristics, very high $\mathrm{S} / \mathrm{N}$, high-resolution spectra of five well-studied

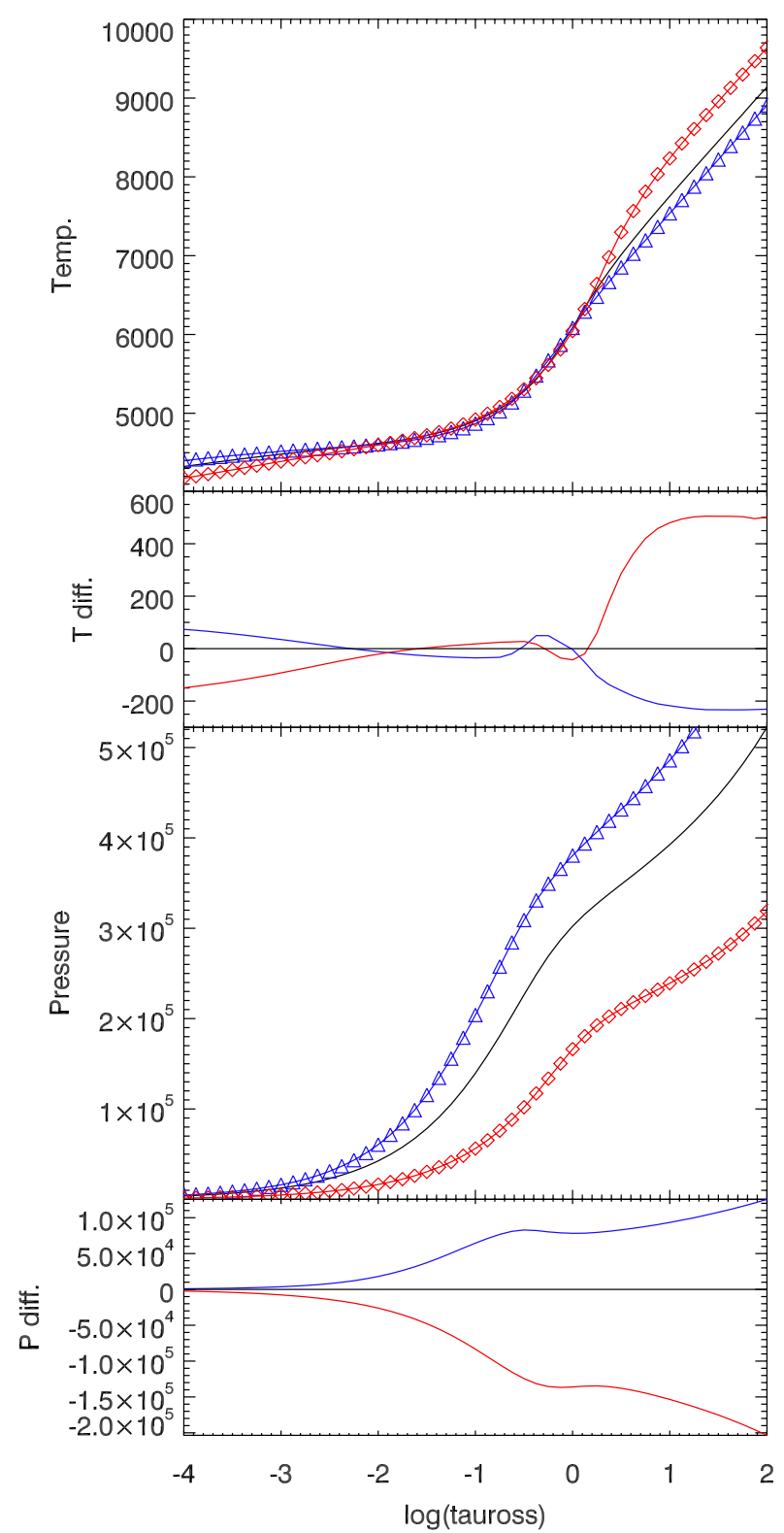

Fig. 4. Comparison of Atlas 12 atmosphere models with $T_{\text {eff }}=5600$ $\log g=4.5, V_{\text {turb }}=1.0,[\alpha / \mathrm{Fe}]=+0.4$ and $[\mathrm{Fe} / \mathrm{H}]=-2.5$ (blue line and triangles), $[\mathrm{Fe} / \mathrm{H}]=-1.5$ (black line), and $[\mathrm{Fe} / \mathrm{H}]=-0.5$ (red line and diamonds). Top to bottom: temperature for the three models, temperature difference $($ red line, $[\mathrm{Fe} / \mathrm{H}]=-0.5-[\mathrm{Fe} / \mathrm{H}]=-1.5$; blue line, $[\mathrm{Fe} / \mathrm{H}]=-2.5-[\mathrm{Fe} / \mathrm{H}]=-1.5)$, pressure in $\mathrm{g} \mathrm{cm}^{-2}$, pressure difference, all plotted vs. $\tau_{\text {ross }}$.

stars have been noise-injected at typical real world values and analyzed using synthetic grids and feature lists appropriate for the four main broad spectra type: metal rich dwarf/subgiant stars (the Sun, see Sect. 6.1), metal poor dwarf/subgiants (HD 126681, Sect. 6.3, and HD 140283, Sect. 6.4), metal rich giants (Arcturus, Sect. 6.2), and metal poor giants (HD 26297, Sect. 6.5). Given the coverage provided by the available synthetic grid, we performed the analysis has been using ranges roughly corresponding to UVES red $580 \mathrm{~nm}$ setting, i.e., two frames covering 480 to $580 \mathrm{~nm}$, and 580 to $680 \mathrm{~nm}$. For each star, Tables 3 and 4 report the final determined parameters, plus detailed abundances for a few chemical species, along with the number of lines used and line-to-line scatter, when more than one line was measured. 
Table 3. Determined abundances for Sun, Arcturus, and HD 126681.

\begin{tabular}{|c|c|c|c|c|c|c|c|c|c|}
\hline & \multicolumn{3}{|c|}{$\begin{array}{l}\text { Sun: } T_{\text {eff }}=5794, \log g=4.55 \\
V_{\text {turb }}=1.07,[\alpha / \mathrm{Fe}]=0.05\end{array}$} & \multicolumn{3}{|c|}{$\begin{array}{l}\text { Arcturus: } T_{\text {eff }}=4293, \log g=1.84 \\
V_{\text {turb }}=1.44,[\alpha / \mathrm{Fe}]=0.19\end{array}$} & \multicolumn{3}{|c|}{$\begin{array}{l}\text { HD 126681: } T_{\text {eff }}=5530, \log g=4.41, \\
V_{\text {turb }}=0.50,[\alpha / \mathrm{Fe}]=0.38\end{array}$} \\
\hline & $\begin{array}{l}\text { Number of } \\
\text { features }\end{array}$ & $\begin{array}{l}{[\mathrm{Fe} / \mathrm{H}] \text { or }} \\
{[\mathrm{X} / \mathrm{Fe}]^{a}}\end{array}$ & $\sigma$ & $\begin{array}{c}\text { Number of } \\
\text { features }\end{array}$ & $\begin{array}{l}{[\mathrm{Fe} / \mathrm{H}] \text { or }} \\
{[\mathrm{X} / \mathrm{Fe}]^{a}}\end{array}$ & $\sigma$ & $\begin{array}{c}\text { Number of } \\
\text { features }\end{array}$ & $\begin{array}{l}{[\mathrm{Fe} / \mathrm{H}] \text { or }} \\
{[\mathrm{X} / \mathrm{Fe}]^{a}}\end{array}$ & $\sigma$ \\
\hline $\mathrm{Fe} I$ & 84 & -0.06 & 0.059 & 59 & -0.44 & 0.073 & 116 & -1.24 & 0.087 \\
\hline Fe II & 14 & -0.05 & 0.094 & 10 & -0.44 & 0.083 & 14 & -1.23 & 0.069 \\
\hline $\mathrm{Na}$ I & 3 & 0.06 & 0.069 & 1 & 0.01 & - & 4 & -0.13 & 0.140 \\
\hline Mg I & - & - & - & - & - & - & 1 & 0.60 & - \\
\hline $\mathrm{Al} \mathrm{I}$ & 3 & -0.04 & 0.073 & 2 & 0.13 & 0.177 & 1 & 0.18 & - \\
\hline Si I & 2 & 0.20 & 0.12 & 8 & 0.33 & 0.123 & 6 & 0.35 & 0.096 \\
\hline Si II & - & - & - & 2 & 0.34 & 0.239 & 1 & 0.57 & - \\
\hline $\mathrm{Ca} \mathrm{I}$ & 8 & 0.02 & 0.074 & 1 & 0.05 & - & 10 & 0.40 & 0.093 \\
\hline Ti I & 23 & 0.04 & 0.086 & 4 & 0.20 & 0.100 & 22 & 0.31 & 0.120 \\
\hline Ti II & 9 & 0.11 & 0.222 & 2 & 0.23 & 0.157 & 10 & 0.32 & 0.250 \\
\hline Ni I & 18 & 0.02 & 0.153 & 6 & 0.02 & 0.126 & 15 & -0.03 & 0.172 \\
\hline
\end{tabular}

Notes. ${ }^{(a)}$ We use $[\mathrm{X} / \mathrm{Fe} \mathrm{I}]$ for neutral species, $[\mathrm{X} / \mathrm{Fe} \mathrm{II}]$ for ionized. The parameter $\sigma$ is line-to-line scatter for Fe, propagated with corresponding $\sigma_{[\mathrm{Fe} / \mathrm{H}]}$ for $[\mathrm{X} / \mathrm{Fe}]$.

Table 4. Determined abundances for HD 140283 with fixed and free $T_{\text {eff }}$, and HD 26297.

\begin{tabular}{|c|c|c|c|c|c|c|c|c|c|}
\hline & \multicolumn{3}{|c|}{$\begin{array}{l}\text { HD } 140283^{a}: T_{\text {eff }}=5777, \log g=3.32 \\
V_{\text {turb }}=1.22,[\alpha / \mathrm{Fe}]=0.26\end{array}$} & \multicolumn{3}{|c|}{$\begin{array}{l}\text { HD } 140283^{b}: T_{\text {eff }}=5506, \log g=2.85 \\
V_{\text {turb }}=1.33,[\alpha / \mathrm{Fe}]=0.35\end{array}$} & \multicolumn{3}{|c|}{$\begin{array}{l}\text { HD 26297: } T_{\text {eff }}=4458, \log g=1.06, \\
V_{\text {turb }}=1.76,[\alpha / \mathrm{Fe}]=0.36\end{array}$} \\
\hline & $\begin{array}{c}\text { Number of } \\
\text { features }\end{array}$ & $\begin{array}{c}{[\mathrm{Fe} / \mathrm{H}] \text { or }} \\
{[\mathrm{X} / \mathrm{Fe}]^{c}}\end{array}$ & $\sigma$ & $\begin{array}{c}\text { Number of } \\
\text { features }\end{array}$ & $\begin{array}{l}{[\mathrm{Fe} / \mathrm{H}] \text { or }} \\
{[\mathrm{X} / \mathrm{Fe}]^{(\mathrm{c})}}\end{array}$ & $\sigma$ & $\begin{array}{c}\text { Number of } \\
\text { features }\end{array}$ & $\begin{array}{l}{[\mathrm{Fe} / \mathrm{H}] \text { or }} \\
{[\mathrm{X} / \mathrm{Fe}]^{c}}\end{array}$ & $\sigma$ \\
\hline $\mathrm{Fe} I$ & 41 & -2.64 & 0.086 & 41 & -2.87 & 0.041 & 85 & -1.83 & 0.070 \\
\hline Fe II & 5 & -2.64 & 0.056 & 5 & -2.86 & 0.062 & 9 & -1.83 & 0.082 \\
\hline $\mathrm{Na} I$ & - & - & - & - & - & - & 3 & -0.41 & 0.102 \\
\hline Mg I & 1 & 0.49 & - & 1 & 0.57 & - & 1 & 0.53 & - \\
\hline $\mathrm{Al} \mathrm{I}$ & - & - & - & - & - & - & 1 & -0.06 & - \\
\hline Si I & - & - & - & - & - & - & 6 & 0.26 & 0.077 \\
\hline $\mathrm{Ca} \mathrm{I}$ & 6 & 0.26 & 0.093 & 6 & 0.35 & 0.044 & 9 & 0.28 & 0.075 \\
\hline Ti I & 4 & 0.40 & 0.087 & 4 & 0.33 & 0.043 & 16 & 0.22 & 0.097 \\
\hline Ti II & 4 & 0.28 & 0.182 & 4 & 0.32 & 0.261 & 5 & 0.23 & 0.356 \\
\hline Ni I & 2 & 0.11 & 0.243 & 2 & 0.11 & 0.155 & 10 & -0.11 & 0.101 \\
\hline
\end{tabular}

Notes. ${ }^{(a)}$ Effective temperature kept fixed at $5777 \mathrm{~K} ;{ }^{(b)}$ effective temperature determined by MyGIsFOS; ${ }^{(c)}$ [X/Fe I] for neutral species, [X/Fe II] for ionized. $\sigma$ is line-to-line scatter for Fe, propagated with corresponding $\sigma_{[\mathrm{Fe} / \mathrm{H}]}$ for $[\mathrm{X} / \mathrm{Fe}]$.

\subsection{The Sun}

Diagnostic plots for the Sun are shown in Fig. 5, and abundances are listed in Table 3. A high $\mathrm{S} / \mathrm{N}(>100)$ spectrum of Ceres was acquired (on 18/01/2008, UT 18:00:56, $900 \mathrm{~s}$ exposure) at OHP/SOPHIE (Bouchy \& Sophie Team 2006; Perruchot et al. 2008 ) in high efficiency mode (resolution of $7.4 \mathrm{~km} \mathrm{~s}^{-1}$ ), and was degraded to UVES-like sampling $(\sim 0.0025 \mathrm{~nm}$ per pixel) and noise-injected to $S / N=50$. Derived parameters $\left(T_{\text {eff }}=\right.$ $5794, \log g=4.55, V_{\text {turb }}=1.07 \mathrm{~km} \mathrm{~s}^{-1},[\alpha / \mathrm{Fe}]=0.05,[\mathrm{Fe} / \mathrm{H}]=$ $-0.06 \pm 0.059$ over $84 \mathrm{Fe}$ I features) are in excellent agreement with the Sun's effective temperature and gravity $\left(T_{\mathrm{eff}}=\right.$ $5777 \mathrm{~K}, \log g=4.44$, Cox 2000). While the canonical value of the solar microturbulence for the full disk flux spectrum is $1.35 \mathrm{~km} \mathrm{~s}^{-1}$ (Holweger et al. 1978), we note that an analysis conducted at lower resolution and based on an ATLAS 9 model derives $0.99 \mathrm{~km} \mathrm{~s}^{-1}$ (Meléndez et al. 2012). The difference from the established values are well within the dispersion of random $S / N=50$ noise injections, as presented below in 7.2.

\subsection{Arcturus}

The UVES POP (Paranal Observatory project, Bagnulo et al. 2003) spectrum for Arcturus derived with the Red UVES arm and $580 \mathrm{~nm}$ setting was convolved with a Gaussian to produce a spectrum with final resolution of $7.0 \mathrm{~km} \mathrm{~s}^{-1}$, then noise-injected at $S / N=50$. Diagnostic plots are shown in Fig. 6, and abundances are listed in Table 3. The derived parameters $\left(T_{\mathrm{eff}}=\right.$ $4293, \log g=1.84, V_{\mathrm{turb}}=1.44 \mathrm{~km} \mathrm{~s}^{-1},[\alpha / \mathrm{Fe}]=0.19,[\mathrm{Fe} / \mathrm{H}]=$ $-0.44 \pm 0.073$, over $59 \mathrm{Fe}$ I features) are in excellent agreement with the values presented in Koch \& McWilliam (2008) $\left(T_{\text {eff }}=\right.$ $\left.4290, \log g=1.64, V_{\text {turb }}=1.54,[\mathrm{Fe} / \mathrm{H}]=-0.49\right)$.

\section{3. $H D 126681$}

The star HD 126681 is a good example of a moderately metalpoor dwarf star. A traditional fully spectroscopic parameter determination for this star, based on high resolution and $\mathrm{S} / \mathrm{N}$ HARPS spectra, is presented in Sousa et al. (2011). It is the 

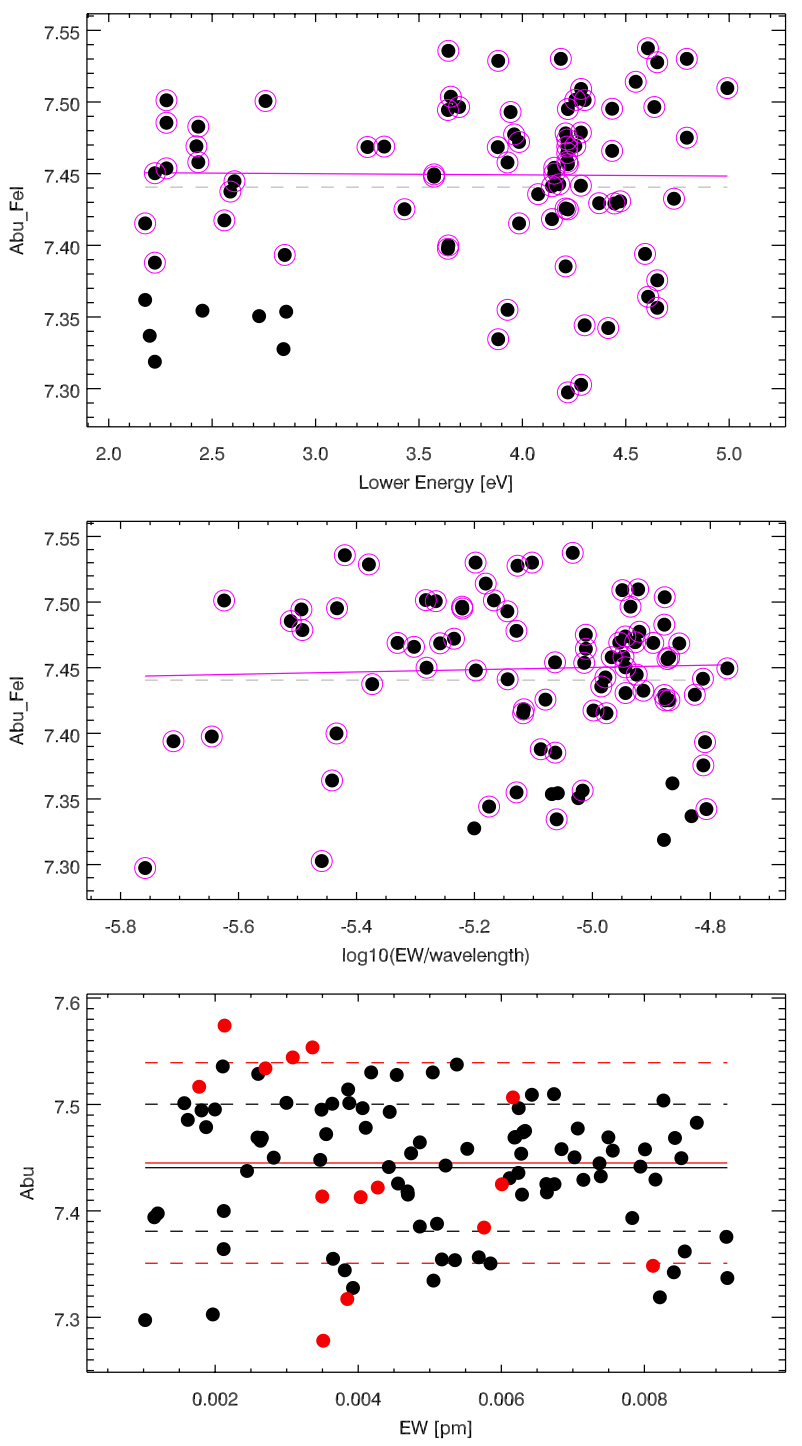

Fig. 5. Diagnostic plots for the determination of atmospheric parameters for the Sun. Upper panel: Fe I lines abundances vs. their lower energy and linear fit for the determination of $T_{\text {eff }}$. Gray dashed line: average Fe I abundance. The magenta line is the linear fit, magentacircled points are the ones considered in the fit. The LEAS is $-8.0 \times$ $10^{-4}$ per eV. Center panel: Fe I lines abundances vs. reduced EW (i.e.,

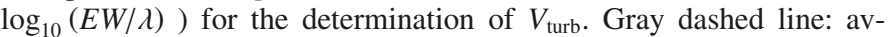
erage Fe I abundance. Magenta symbols and line as in the top panel. Linear fit slope is $8.7 \times 10^{-3}$. Bottom panel: Fe I (black) and Fe II (red) lines abundances for the check of gravity through ionization equilibrium. The continuous lines represent averages, dashed lines $1 \sigma$ intervals. $A(\mathrm{Fe} \mathrm{I})=7.44 \pm 0.06, A(\mathrm{Fe} I \mathrm{II})=7.45 \pm 0.09$.

very type of analysis MyGIsFOS aims to replicate, and leads to values of $T_{\text {eff }}=5561 \pm 68 \mathrm{~K}, \log g=4.71 \pm 0.1, V_{\text {turb }}=$ $0.71 \mathrm{~km} \mathrm{~s}^{-1}$, and $[\mathrm{Fe} / \mathrm{H}]=-1.14 \pm 0.06$. We employed again the UVES POP spectrum, degraded to a resolution of $7.4 \mathrm{~km} \mathrm{~s}^{-1}$, and noise-injected to $S / N=80$. Diagnostic plots for HD 126681 are shown in Fig. 7, and the abundances are listed in Table 3. The derived values $\left(T_{\text {eff }}=5530, \log g=4.41, V_{\text {turb }}=0.50\right.$, $[\alpha / \mathrm{Fe}]=0.38,[\mathrm{Fe} / \mathrm{H}]=-1.24 \pm 0.087$, over 116 features $)$ are in very good agreement with the above stated values. The $[\mathrm{Fe} / \mathrm{H}]$ discrepancy is a bit higher than expected, our metallicity being lower by 0.10 dex. It is worth noting how a change upward of Fe I abundance alone would lead ionization equilibrium to settle to a somewhat higher gravity, as found by Sousa et al. (2011).
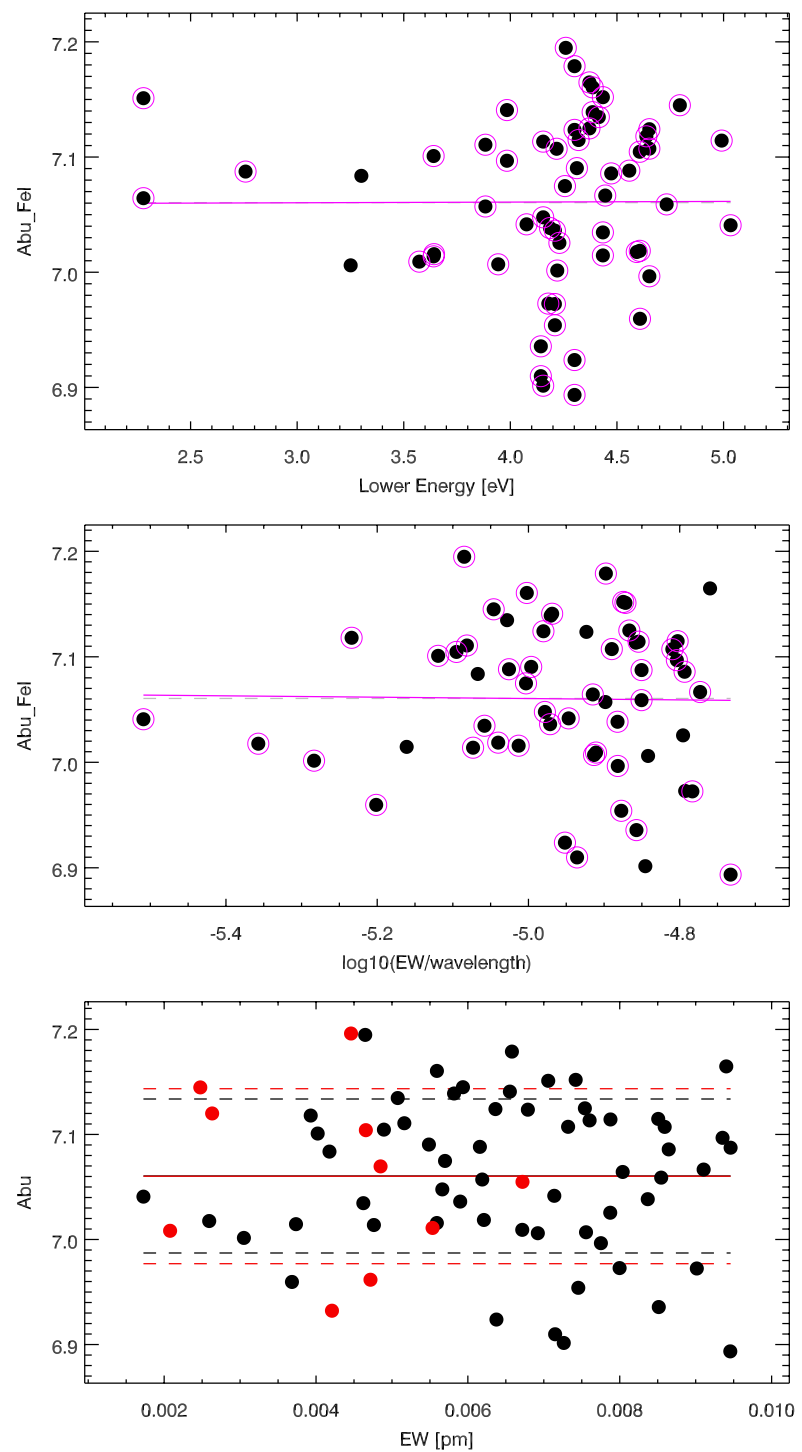

Fig. 6. Same as in Fig. 5 for Arcturus. The LEAS $=0.0006$ per eV. Fe I abundance vs. reduced EW slope $-6.4 \times 10^{-3}$. A(Fe I $)=7.06 \pm$ $0.07, A(\mathrm{Fe}$ II $)=7.06 \pm 0.08$.

We have not further investigated this slight discrepancy, which can stem from a number of differences, including differences in Fe I line data, or the fact that Sousa et al. (2011) use a model grid with a different set of opacities and the overshooting option switched on. The difference between overshooting and non overshooting ATLAS models can account for a 0.1 dex difference.

\subsection{HD 140283}

The star HD 140283 is the prototypical metal-poor star (Chamberlain \& Aller 1951), very often employed as a reference for stars in this metallicity range (recently, Hosford et al. 2009, 2010; Casagrande et al. 2010; Bergemann et al. 2012, among others). Based on recent Hubble Space Telescope (HST) observations, Bond et al. (2013) estimated the distance of HD 140283, and consequently estimated its age at $14.46 \pm 0.8$ Gyr. Hosford et al. (2009) noticed how attempting to constrain at the same time ionization and excitation equilibrium led to a relatively low temperature $(5573 \mathrm{~K})$ and consequently to a low gravity incompatible with the known HIPPARCos-based luminosity. The infrared flux method (IRFM) based temperature $(5691 \mathrm{~K}$ 

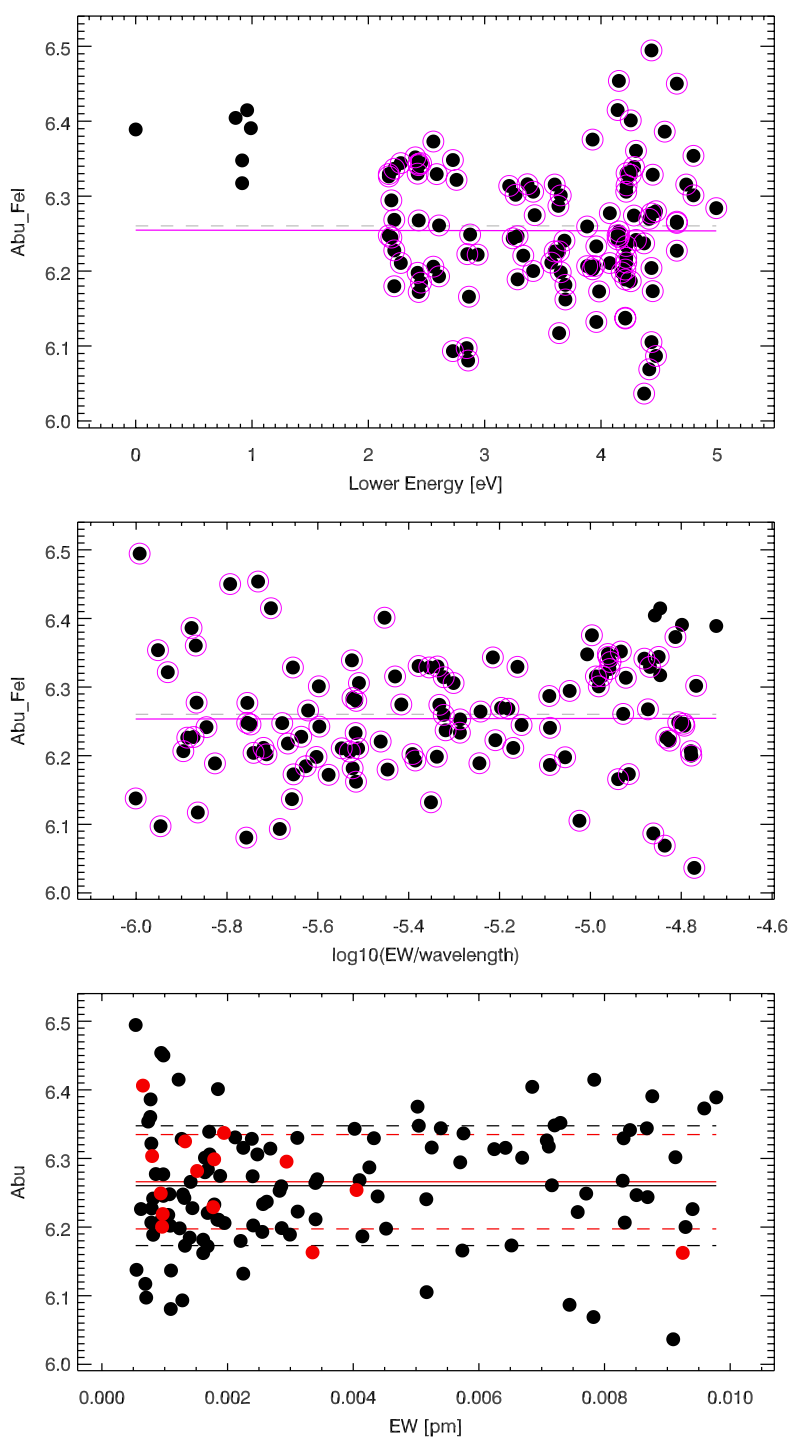

Fig. 7. Same as in Fig. 5 for HD 126681. LEAS $=-0.0002$ per eV. Fe I abundance vs. reduced EW slope $8.0 \times 10^{-4} . A(\mathrm{Fe} \mathrm{I})=6.26 \pm 0.09$, $A(\mathrm{Fe}$ II $)=6.27 \pm 0.07$.

Alonso et al. 1996; 5755 K González Hernández \& Bonifacio 2009; 5777 K Casagrande et al. 2010) delivers a more satisfactory ionization equilibrium when gravity is derived from the HIPPARCOS luminosity, but this is at the expense of inducing a sizeable LEAS (see Tables 2, 3, and Fig. 11 in Bergemann et al. 2012). According to Hosford et al. (2010), treating the line transfer for Fe I lines in NLTE solves this discrepancy and provides an excitation temperature of $5838 \mathrm{~K}$, in reasonable agreement with the IRFM temperatures. Bergemann et al. (2012) disagree with this finding. What is relevant in the present context is that solving for both excitation and ionization equilibria of iron in LTE for HD 140283 leads to low temperature and gravity; Hosford et al. (2009) and Bergemann et al. (2012) agree with this issue.

We employed the UVES POP red arm $580 \mathrm{~nm}$ spectra, without adding instrumental broadening or injecting noise: due to the high resolution of the spectra, stellar rotation and macroturbulence might require a broadening of the grid above what is necessary to take the instrumental resolution into account. We adjusted the grid broadening until we obtained satisfactory fits on several isolated lines. The final grid broadening employed was $5.5 \mathrm{~km} \mathrm{~s}^{-1}$. We ran MyGIsFOS on this star twice, once leaving
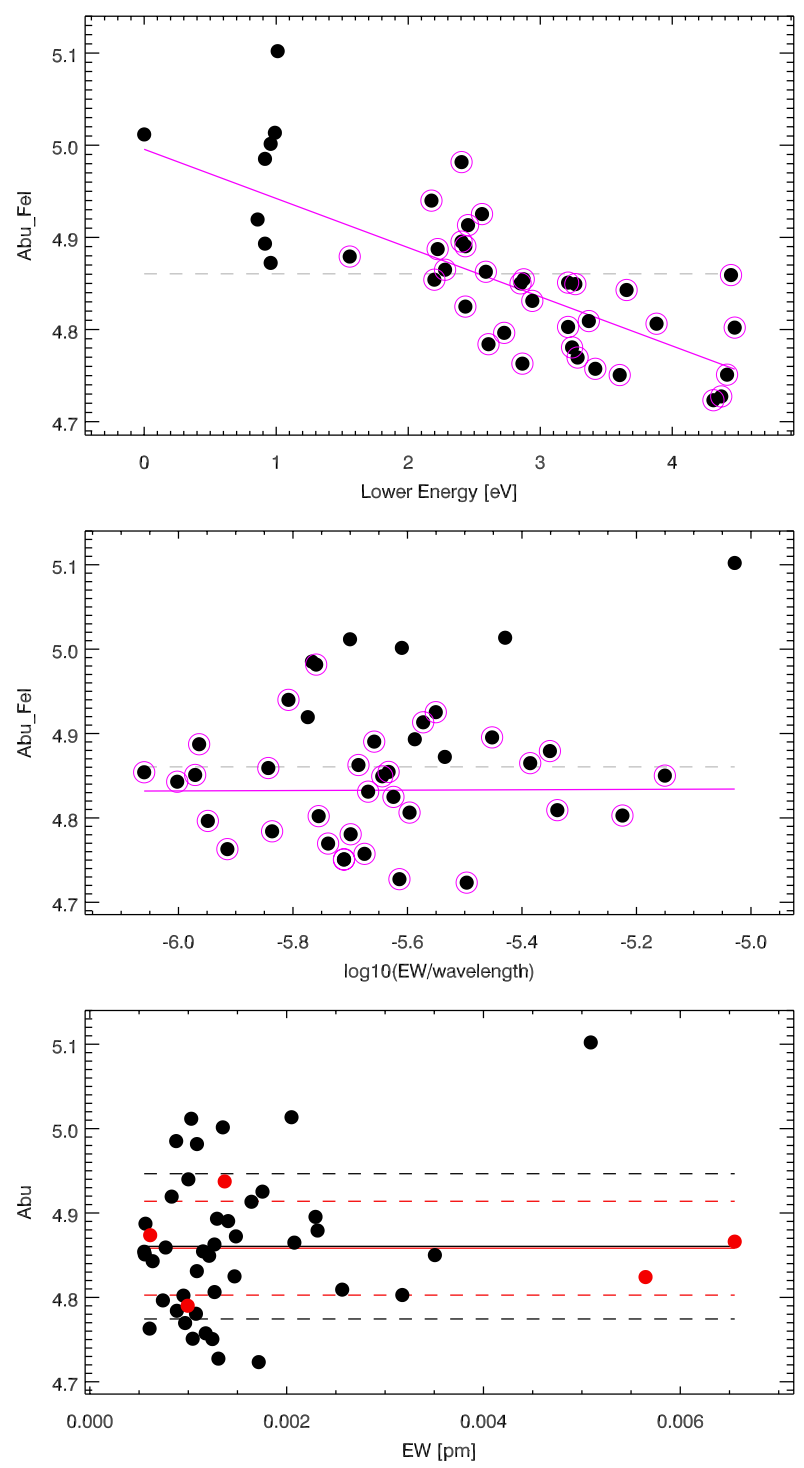

Fig. 8. Same as in Fig. 5 for HD 140283 with the temperature locked at $T_{\text {eff }}=5777 \mathrm{~K} . \mathrm{LEAS}=-0.0534$ per $\mathrm{eV}$. The Fe I abundance vs. reduced $\mathrm{EW}$ slope is $2.3 \times 10^{-3} . A(\mathrm{Fe} \mathrm{I})=4.86 \pm 0.09, A(\mathrm{Fe} \mathrm{II})=4.86 \pm 0.06$.

the code free to constrain all the parameters, and once locking $T_{\text {eff }}$ to $5777 \mathrm{~K}$. Diagnostic plots for HD 140283 are shown in Figs. 8 and 9, and the abundances are listed in Table 4. When leaving MyGIsFOS free to set $T_{\text {eff }}$, it falls to $5506 \mathrm{~K}$, reasonably close to the excitation temperature found by Hosford et al. (2009) also driving down $[\mathrm{Fe} / \mathrm{H}]$ to -2.87 , and $\log g$ to 2.86 . When fixing $T_{\text {eff }}$ at $5777 \mathrm{~K}$, instead, the derived gravity (3.32) is in reasonable agreement with the HIPPARCos value (3.73), and $V_{\text {turb }}$ is also very close to the value derived in Bergemann et al. (2012) in the LTE case (1.22 $\mathrm{km} \mathrm{s}^{-1}$ vs. 1.27). However, we derive a metallicity lower by about $0.22 \mathrm{dex}([\mathrm{Fe} / \mathrm{H}]=-2.64$ vs. -2.42$)$.

\subsection{HD 26297}

The star HD 26297 is a moderately metal-poor giant star. A spectroscopic abundance analysis similar to the one MyGIsFOS performs is presented in Fulbright (2000) $\left(T_{\mathrm{eff}}=4500 \mathrm{~K}, \log g=\right.$ $1.2,[\mathrm{Fe} / \mathrm{H}]=-1.72)$, while Gratton et al. (2000) obtain similar values $\left(T_{\text {eff }}=4450 \mathrm{~K}, \log g=1.18,[\mathrm{Fe} / \mathrm{H}]=-1.68\right.$, 

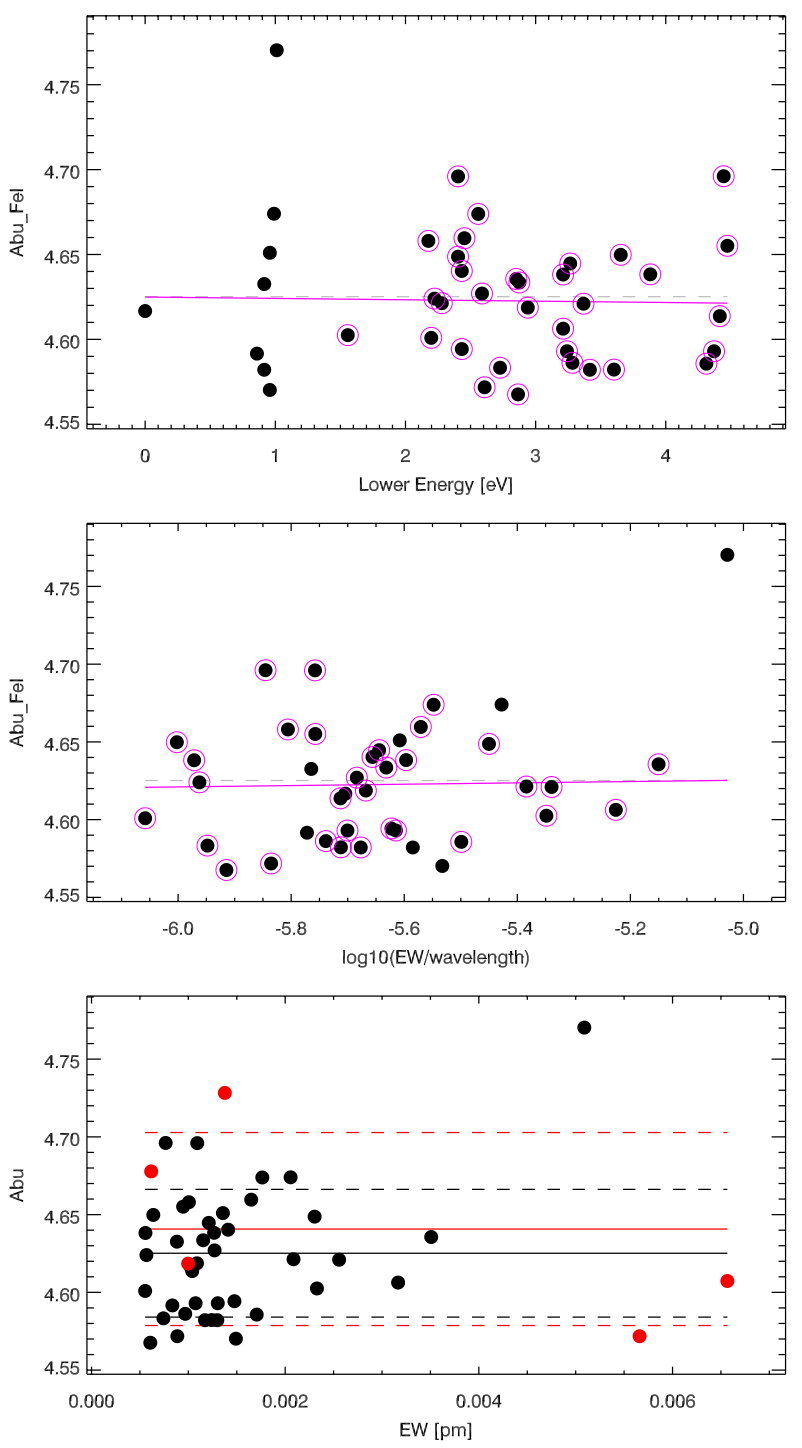

Fig. 9. Same as in Fig. 5 for HD 140283 with temperature iterated. LEAS $=-0.0008$ per eV. The Fe I abundance vs. reduced EW slope is $4.3 \times 10^{-3} . A(\mathrm{Fe} \mathrm{I})=4.63 \pm 0.04, A(\mathrm{Fe}$ II $)=4.64 \pm 0.06$.

$V_{\text {turb }}=1.62 \mathrm{~km} \mathrm{~s}^{-1}$ ) but derive temperature and gravity from photometric calibrations ( $\mathrm{Fe}$ ionization equilibrium is very well satisfied for this star). A more recent analysis, presented in Prugniel et al. (2011), uses a $\chi^{2}$ minimization algorithm instead to fit the target spectrum against the empirical ELODIE library (see Wu et al. 2011), and delivers $T_{\text {eff }}=4479 \mathrm{~K}, \log g=1.05$, $[\mathrm{Fe} / \mathrm{H}]=-1.78$.

We downloaded archival UVES red 580 spectra for HD 26297 (taken on 20/11/2004, 01:39:42 UT, prog. id 074.B0639). The data were taken with slit width of $0.9^{\prime \prime}$ and have a good S/N (>100). No additional noise was injected. They were analyzed by broadening the synthetic grid to $7.5 \mathrm{~km} \mathrm{~s}^{-1}$. Diagnostic plots are shown in Fig. 10, and the abundances are listed in Table 4. We derive $T_{\text {eff }}=4458, \log g=1.06, V_{\text {turb }}=$ $1.76,[\alpha / \mathrm{Fe}]=0.36,[\mathrm{Fe} / \mathrm{H}]=-1.83 \pm 0.07$, over $83 \mathrm{Fe}$ I lines, in excellent agreement with all the cited sources.

\section{Monte Carlo simulations}

One attractive possibility opened by fully automated abundance analysis codes such as MyGIsFOS is to comprehensively assess
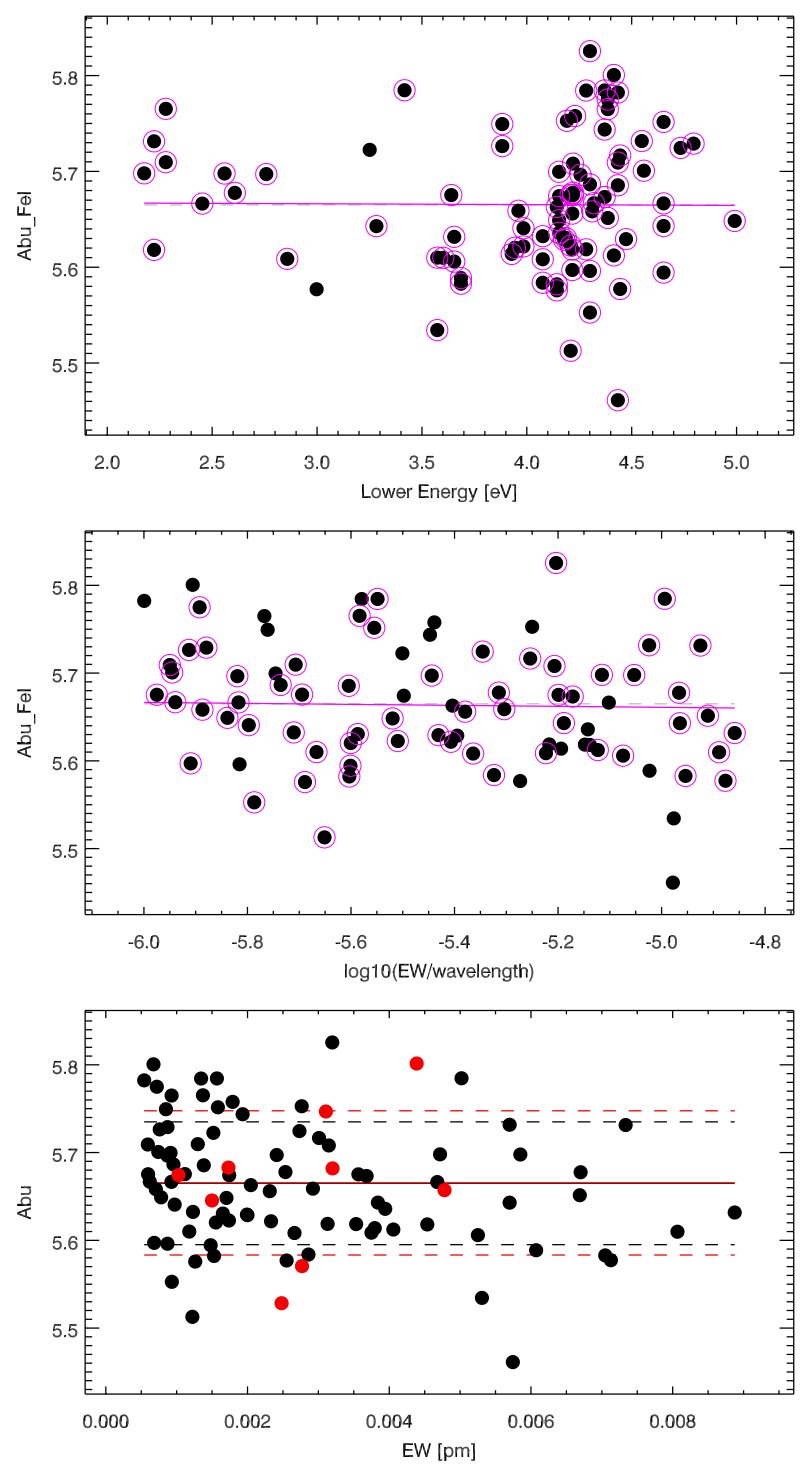

Fig. 10. Same as in Fig. 5 for HD 26297. LEAS $=0.0009$ per eV. The Fe I abundance vs. reduced EW slope is $-5.6 \times 10^{-3} . A(\mathrm{Fe} \mathrm{I})=5.67 \pm$ $0.07, A(\mathrm{Fe}$ II $)=5.67 \pm 0.08$.

the error budget by means of Monte Carlo simulations, since large number of test events can be processed through the code in very little time.

\subsection{Impact of $\mathrm{S} / \mathrm{N}$ : internal errors}

To assess the size of MyGIsFOS internal errors, as well as the extent to which the code is affected by spectrum noise, we employed a synthetic spectrum extracted from the MPD grid at $T_{\text {eff }}=5400 \mathrm{~K}, \log g=4.5, V_{\text {turb }}=1.0 \mathrm{~km} \mathrm{~s}^{-1},[\mathrm{Fe} / \mathrm{H}]=-1.0$, $[\alpha / \mathrm{Fe}]=+0.4$, and processed it to MyGIsFOS after broadening it to a resolution of $7.4 \mathrm{~km} \mathrm{~s}^{-1}$, and resampling it to typical UVES pixel size. We first processed it without injecting noise to ensure the absence of systematics, and subsequently produced 1000 independent Poisson noise realizations at $S / N=80,50,20$, for a total of 3000 noise realizations, which were fed to MyGIsFOS, set to derive $T_{\text {eff }}, \log g, V_{\text {turb }},[\mathrm{Fe} / \mathrm{H}]$, and $[\alpha / \mathrm{Fe}]$. The results of the noiseless test, as well as derived average values and $1 \sigma$ dispersions for the noise-injection Monte Carlo tests are listed in Table 5. 
Table 5. Average parameter values and $1 \sigma$ intervals for the Monte Carlo tests described in Sect. 7.

\begin{tabular}{lrrrrrrrrr}
\hline \hline Test type & $\begin{array}{r}T_{\text {eff }} \\
\mathrm{K}\end{array}$ & $\sigma$ & $\begin{array}{r}\log g \\
\mathrm{~cm} \mathrm{~s}^{-1}\end{array}$ & $\sigma$ & {$[\mathrm{Fe} / \mathrm{H}]$} & $\sigma$ & $\begin{array}{r}V_{\text {turb }} \\
\mathrm{km} \mathrm{s}^{-1}\end{array}$ \\
\hline Synthetic noiseless & 5403 & - & 4.51 & - & -1.00 & - & 0.97 & - \\
\hline Synthetic $S / N=80$ & 5409 & 31 & 4.51 & 0.06 & -1.01 & 0.02 & 1.05 & 0.07 \\
Synthetic $S / N=50$ & 5419 & 36 & 4.54 & 0.08 & -1.00 & 0.03 & 1.04 & 0.09 \\
Synthetic $S / N=20$ & 5461 & 80 & 4.57 & 0.18 & -0.95 & 0.06 & 0.94 & 0.22 \\
\hline HD 126681 $S / N=80$ & 5536 & 36 & 4.42 & 0.07 & -1.24 & 0.03 & 0.58 & 0.16 \\
HD 126681 $S / N=50$ & 5499 & 46 & 4.34 & 0.10 & -1.24 & 0.03 & 0.47 & 0.19 \\
HD 126681 $S / N=20$ & 5422 & 87 & 4.10 & 0.20 & -1.22 & 0.07 & 0.39 & 0.28 \\
\hline HD 126681 fixed $T_{\text {eff }}, S / N=80$ & $\mathbf{5 5 5 2}$ & $\mathbf{1 5 0}$ & 4.43 & 0.29 & -1.24 & 0.12 & 0.77 & 0.22 \\
\hline
\end{tabular}

Notes. In order, the result of the analysis of the noiseless synthetic spectrum, of the three 1000 noise realization runs on the synthetic (for both see 7.1), of the three 1000 noise realization runs on HD 126681, and of the "photometric $T_{\text {eff }}$ " run on HD 126681 (for both see 7.2). In the last row, $T_{\text {eff }}$ and its $\sigma$ are in boldface to indicate they are not results, but the fixed input $T_{\text {eff }}$ distribution.
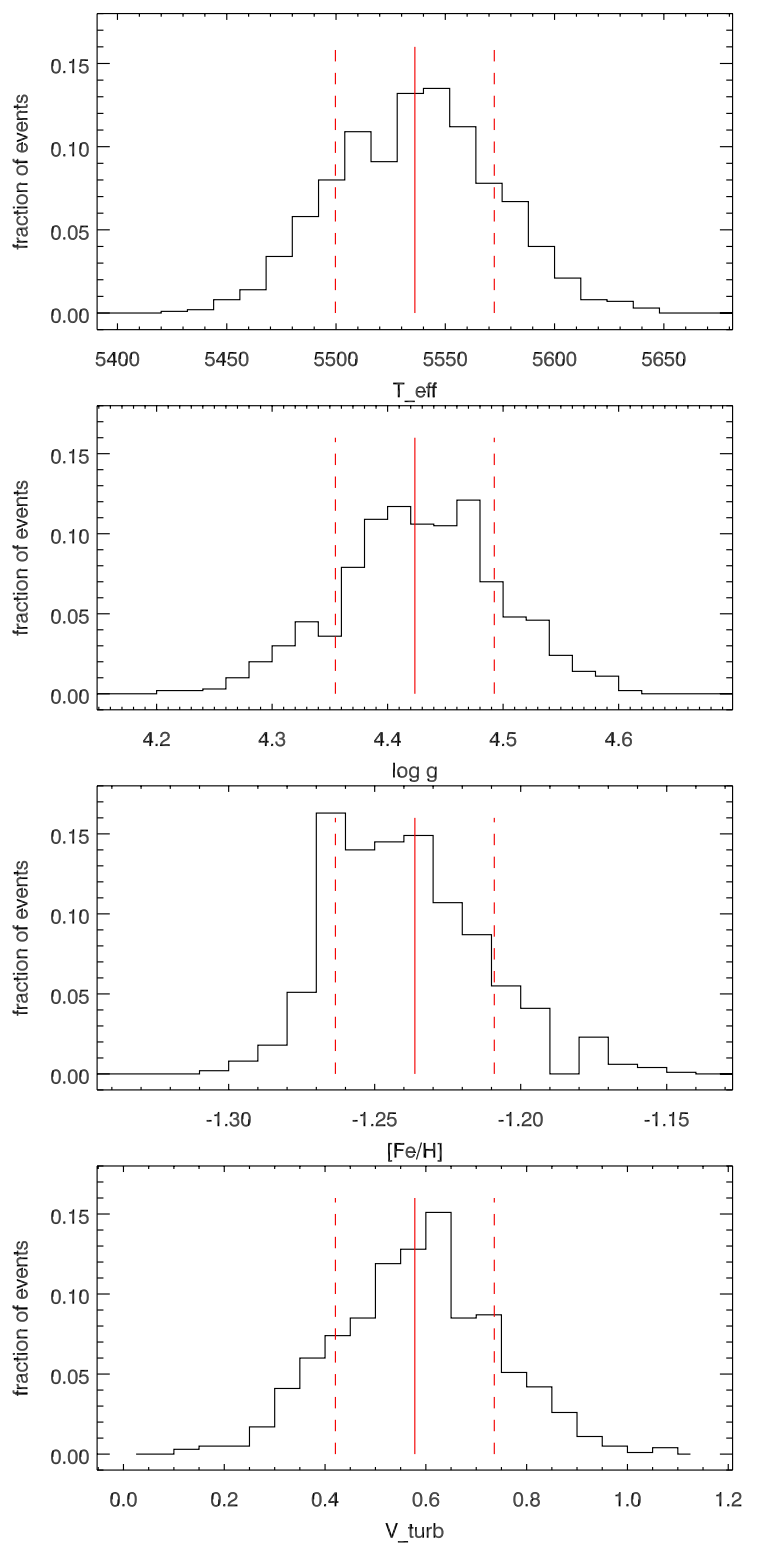

Fig. 11. Histograms of MyGIsFOS parameter determination output in a Monte Carlo simulation of 1000-events noise injection at $S / N=80$ on a spectrum of HD 126681. Top to bottom: determined $T_{\text {eff }}$ (mean and $\sigma$, continuous and dashed red lines, respectively, all values in Table 5), $\log g,[\mathrm{Fe} / \mathrm{H}], V_{\text {turb }}$.
It is worth noticing how the errors displayed by MyGIsFOS in the synthetic test closely match the ones in the case of HD126681. One might have expected that feeding MyGIsFOS a spectrum of the same grid used for the analysis would have led to the code snapping on the right parameters, leading to lowerthan-realistic errors. However, MyGIsFOS only compares the grid and the observed spectrum on a line-by-line basis, and parameters are all derived by indirect methods. Moreover, the initial temperature scan is purposefully performed at steps different from the grid step, so that the comparison is always performed away from gridpoints, removing any grid snapping effect.

\subsection{Impact of $S / N: H D 126681$}

To assess the impact of external error sources we have repeated the above described test on the very high $\mathrm{S} / \mathrm{N}$ spectrum of HD126681 described in Sect. 6.3. Again, 1000 independent Poisson noise realizations were produced for $S / N=80,50$, 20 each. Derived average values and $1 \sigma$ dispersions are listed in Table 5, the histograms of the parameters are presented in Figs. 11 through 13.

MyGIsFOS is not allowed to try to bring stellar parameters to convergence indefinitely. After a set number of cycles it stops, declares the star non-converging and moves on to the next one. It is worth noticing here that, while $S / N=80$ and 50 Monte Carlo tests had every single star reaching satisfactory convergence, in the $S / N=20$ case convergence was reached in 943 over 1000 realizations (a failure rate of 5.7\%). Unsurprisingly, at such low $\mathrm{S} / \mathrm{N}$ the difficulty of measuring weak lines, needed especially for $V_{\text {turb }}$ determination, begins to take its toll. Moreover, $V_{\text {turb }}$ is estimated at $0 \mathrm{~km} \mathrm{~s}^{-1}$ for 138 converging stars, a clearly visible peak in Fig. 13. This is just the natural consequence of a $V_{\text {turb }}$ distribution centered at $0.39 \mathrm{~km} \mathrm{~s}^{-1}$ with a $\sigma$ of 0.28 : in a relevant number of stars the specific set of Fe I lines used would push for a negative $V_{\text {turb }}$, which MyGIsFOS forbids as unphysical.

The presented test is a simplified one and the resulting uncertainties are possibly somewhat underestimated. In particular, the injection of Poisson noise is a somewhat optimistic way to simulate real observed spectrum noise degradation, since it lacks a number of realistic outstanding defects real, low $\mathrm{S} / \mathrm{N}$ spectra present (cosmic ray hits, poor order tracing in low $\mathrm{S} / \mathrm{N}$ cases, increased noise at order merging points...). Also, noise was injected here to produce constant $\mathrm{S} / \mathrm{N}$ through the range, which is not the case in practice, often with lower $\mathrm{S} / \mathrm{N}$ in the bluer part of the range, where most of the atomic lines are concentrated. 
L. Sbordone et al.: A code for automatic abundance analysis
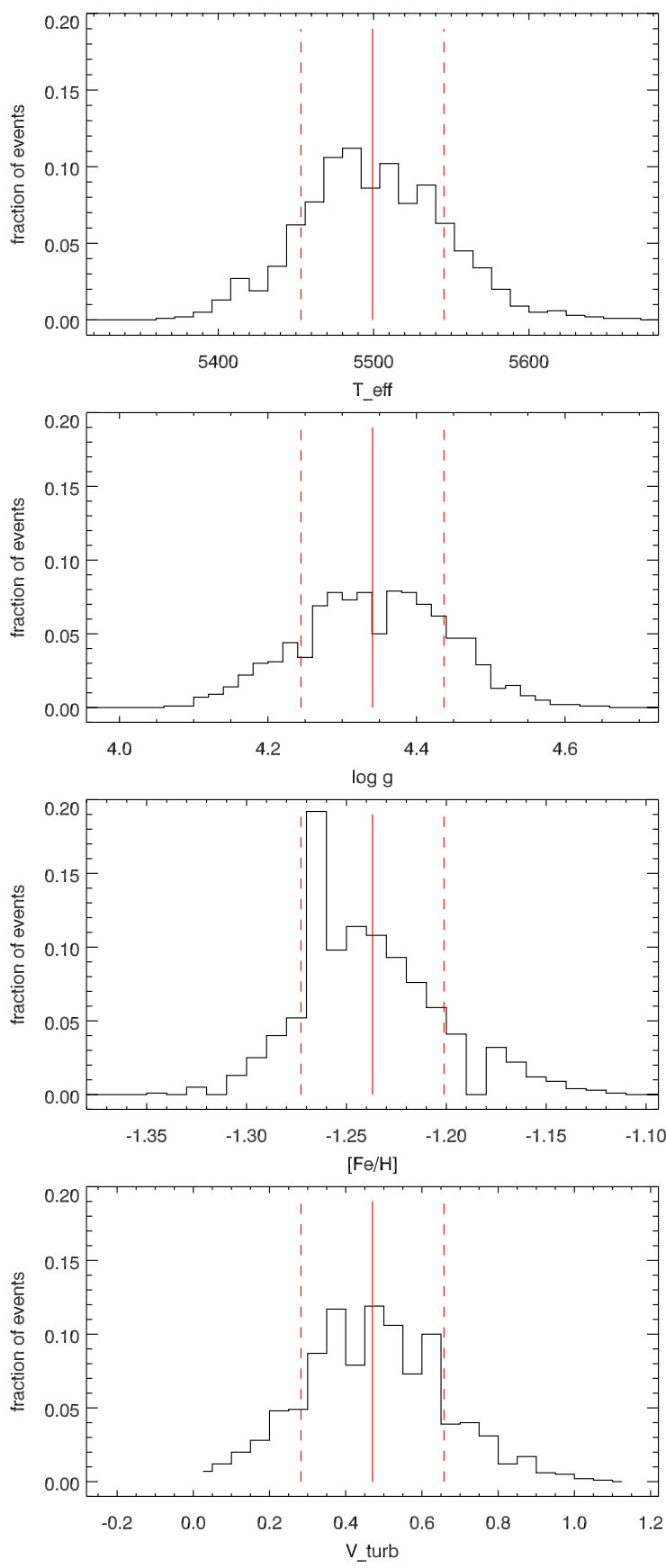

Fig. 12. As in Fig. 11 but now with $S / N=50$.

\subsection{Impact of predetermined parameters: photometric $T_{\text {eff }}$}

Most abundance analysis works typically assess the impact of errors on atmospheric parameters on derived abundances by assuming said errors are independent. This is often performed by picking a representative star in the sample, and repeating the abundance determination by varying one parameter at a time by an amount considered to be the typical uncertainty on that parameter. This quick way of estimating parameter uncertainties is, however, conceptually flawed in the sense that atmospheric parameters correlate in a fashion that is, ultimately, dependent on the specific way they are derived. For instance, if gravity is determined from Fe I-Fe II ionization equilibrium, varying $T_{\text {eff }}$ by $150 \mathrm{~K}$ will lead to a different gravity too: repeating the analysis with a different temperature but the same gravity will not be
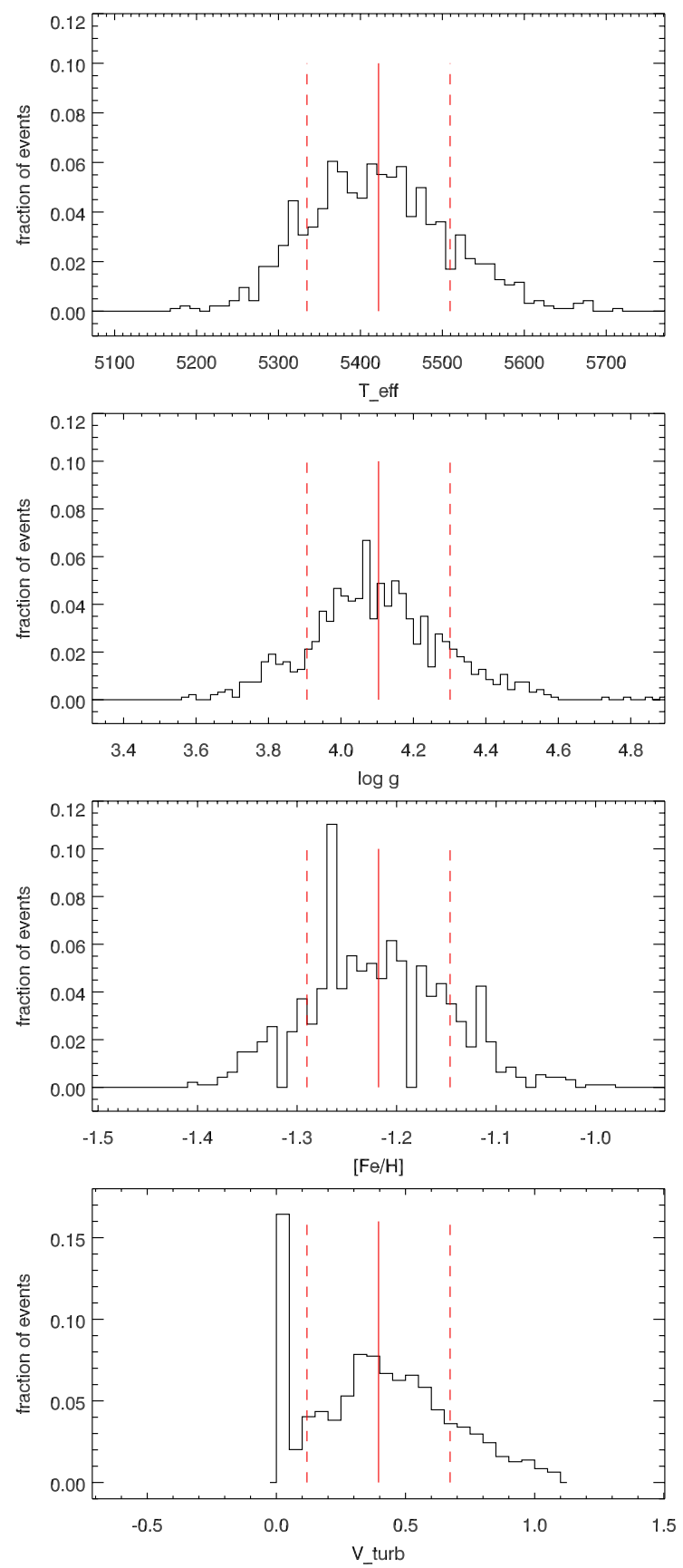

Fig. 13. As in Fig. 11, but now with $S / N=20$.

representative of which effect a $150 \mathrm{~K} T_{\text {eff }}$ error would have in a real world case.

A fairly common case in which such issue is of importance is the one in which effective temperatures are derived independently from the spectra, e.g., from photometry. In this case, errors in the other parameters cascade from whatever error is made in $T_{\text {eff }}$, in a systematic way. MyGIsFOS allows us to estimate the effect through a Monte Carlo test set. We again constructed a 1000-events test set but, in contrast to what we did in 7.2, we have now analyzed one single realization of $S / N=80$ noise injection, starting from the same HD 126681 high-S/N spectrum. We now constructed the test set by drawing $1000 T_{\text {eff }}$ values from a Gaussian distribution centered at $5552 \mathrm{~K}$ and with a $\sigma$ of $150 \mathrm{~K}$, and having MyGIsFOS run with fixed $T_{\text {eff. }}$ The resulting histograms are shown in Fig. 14, the resulting distributions in 

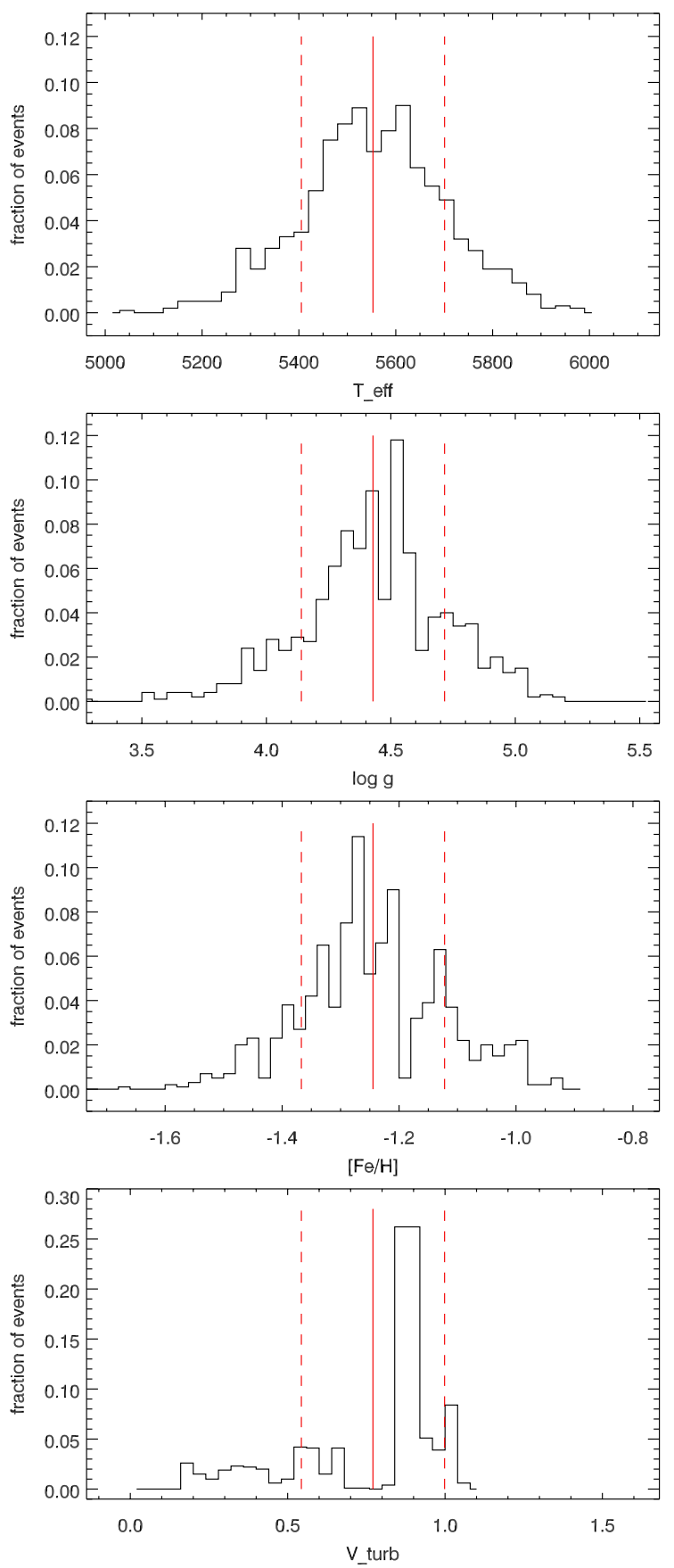

Fig. 14. Histograms of MyGIsFOS parameter determination output in a 1000-events Monte Carlo simulation with fixed $T_{\text {eff }}$, on a $S / N=80$ spectrum of HD 126681. Panels as in Fig. 11.

the other parameters and metallicity are again listed in Table 5. Although we do not present it here, a more detailed speciesby-species error estimate is of course possible, thus allowing to properly assess parameter-related uncertainties on any abundance. The same kind of procedure is, of course, possible when more than one parameter is kept fixed (e.g., the case of photometric $T_{\text {eff }}$ and $\log g$ derived from isochrones).

\section{Performance, system requirements, and availability}

MyGIsFOS is written in Fortran 90 with Intel extensions. So far it has been compiled and run under Linux and Mac OS X.
MyGIsFOS synthetic grids can grow to significant sizes when covering large spectral ranges and a large parameter space. As an example, we consider the grid employed in Sects. 6.3, 6.4, and 7, intended to handle UVES RED 580 spectra of metal poor, cool turn off and dwarf stars. It is composed of two frames (460 to 590 , and 560 to $690 \mathrm{~nm}$ ) synthesized (from ATLAS12 atmosphere models and by using SYNTHE, see Sbordone et al. 2004; Castelli 2005; Kurucz 2005; Sbordone 2005) with velocityequispaced, $R=300000$ sampling. The spectra are computed for 3840 parameter values ${ }^{5}$. The binary packaged grid has a size of about $2.2 \mathrm{~GB}$. The size of the grid sets the main resource requirement, since the grid is twice resident in memory (the master copy read in at the beginning of the run, and the one, smaller, resampled to the observed pixels of the star being currently processed). Also, grid resampling at observed spectrum pixel wavelengths is usually the longest operation in the processing of a star. For both these reasons, it is always advisable to use the smallest desirable grid both in terms of parameter space and spectrum coverage.

As a benchmarking and resource consumption reference, we use typical values for one of the $\mathrm{S} / \mathrm{N}$ Monte Carlo runs described in Sect. 7.2. The run was executed on a quad-core Intel Xeon E31245, 3.3 GHz, machine with $11.6 \mathrm{~GB}$ of RAM, running openSUSE 12.3 with 3.7.10 64 bit kernel. Employing the grid described above in this same section, peak MyGIsFOS memory consumption was $3.5 \mathrm{~GB}$, with an average processing time per star (over 1000 stars) of 119 s, running on a single core ${ }^{6}$. These values are to be considered upper limits. In the described case, the resampling of $\sim 74700$ synthetic grid "pixels" over the $\sim 71000$ observed ones takes somewhat more of $50 \%$ of the total per-star processing time, almost all the rest being taken by the $\sim 280$ parameter-finding iterations needed to determine $T_{\text {eff }}$, $\log g V_{\text {turb }},[\mathrm{Fe} / \mathrm{H}]$, and $[\alpha / \mathrm{Fe}]$. At the other extreme, determining only $[\mathrm{Fe} / \mathrm{H}],[\alpha / \mathrm{Fe}]$, and detailed abundances at predefined atmospheric parameters on data constituted by two FLAMES high-resolution settings takes less than $10 \mathrm{~s}$ per star, thanks equally to the lack of parameter iteration and to the small number of pixels over which grid resampling should be performed.

For the time being, MyGIsFOS is available on a collaboration basis only. A website has been set up to make detailed code documentation available to interested parties, so that they can assess whether it can be of use to them before contacting us.

\section{Conclusions}

We have presented MyGIsFOS, an automatic code for the determination of atmospheric parameters and detailed chemical abundances in cool stars. Replicating as closely as possible a traditional manual abundance analysis technique for these types of stars, MyGIsFOS derives results directly and quickly comparable with well-known analysis techniques. Currently, MyGIsFOS has been employed on the same type of data that are best suited for the manual techniques it replicates, i.e., those characterized by high-resolution and/or broad spectral coverage, such as the ones delivered by UVES, X-shooter (Vernet et al. 2011),

5 Listing start, end, step, and number of steps. $T_{\text {eff }}[\mathrm{K}]: 5000,6000$, 200, 6; $\log g\left[\mathrm{~cm} \mathrm{~s}^{-1}\right]: 3.0,5.0,0.5,5 ; V_{\text {turb }}\left[\mathrm{km} \mathrm{s}^{-1}\right]: 0.0,3.0,1.0,4$; $[\mathrm{Fe} / \mathrm{H}]:-4.0,-0.5,0.5,8 ;[\alpha / \mathrm{Fe}]:-0.4,0.8,0.4,4$.

6 MyGIsFOSis not currently written or compiled to use more than one thread. This would surely speed up the execution significantly, but we do not see it as a priority, since the code is already fast enough that the actual bottleneck is the "human time" to prepare the input and check the results, rather than the processor time. 
HARPS, Sophie (Caffau et al. 2011a; Bonifacio et al. 2012; Caffau et al. 2012, 2013; Duffau et al., in prep.). Its very fast operation (two minutes or less per star on a mainstream personal computer) is useful in a number of circumstances. For instance, it is convenient when analyzing large datasets of (broadly) homogeneous data in the context of large scale spectroscopic surveys, for instance, the Gaia-ESO Public Survey (UVES data, see Gilmore et al. 2012). It can also be used when screening large amounts of low-intermediate resolution spectra for interesting candidates to be followed up. While MyGIsFOS delivers its full capability for high-resolution $(R \geq 25000)$, large spectral coverage data, it can be effectively used to derive global metallicity from low-resolution spectra, once parameters can be inferred from other sources. In this context Abbo (Bonifacio \& Caffau 2003), the code MyGIsFOS has been derived from, has been employed to select extremely metal poor turn off candidates from low-resolution SDSS-SEGUE (York et al. 2000; Yanny et al. 2009) spectra, which were then followed up at high resolution, showing a remarkable selection success rate (e.g., Caffau et al. 2012; Bonifacio et al. 2012; Caffau et al. 2011a), and MyGIsFOS is currently used in the same capacity in the context of the TOPoS ESO Large Program (Turn-Off Primordial Stars, Caffau et al. 2013). It can also be very useful to extend preexisting literature datasets with new data: when adding new stars to pre-existing samples of abundances derived in previous papers, it is usually impractical to reanalize the existing corpus of data, so homogeneity issues arise because of differences, e.g., in atmosphere modeling, or atomic data choices. The very fast processing of MyGIsFOS permits, on the other hand, repeated analysis of the whole sample in a fully homogeneous way every time. As a matter of fact, fully parallel analyses can be conducted, e. g., with different atmospheric parameter choices, with practically no time penalty. Also, MyGIsFOS allows to consistently tackle error propagation through the analysis pipeline by means of Monte Carlo simulations, as presented in Sect. 7.3 for photometric effective temperatures. This is almost never done due to the very high time cost it would usually imply, but it would be of great importance since errors in atmospheric parameters and abundances are, in general, strongly correlated, and method dependent.

Acknowledgements. L.S., E.C., and S.D. aknowledge the support of Sonderforschungsbereich SFB 881 "The Milky Way System" (subprojects A4 and A5) of the German Research Foundation (DFG). P.B. acknowledges support from the Conseil Scientifique de l'Observatoire de Paris and from the Programme National de Cosmologie et Galaxies of the Institut National des Sciences de l'Univers of CNRS. This research has made use of the SIMBAD database, operated at CDS, Strasbourg, France, and of NASA's Astrophysics Data System, and is partly based on data obtained from the ESO Science Archive Facility.

\section{References}

Allende Prieto, C. 2004, Astron. Nachr., 325, 604

Allende Prieto, C., Rebolo, R., García López, R. J., et al. 2000, AJ, 120, 1516

Allende Prieto, C., Sivarani, T., Beers, T. C., et al. 2008, AJ, 136, 2070

Alonso, A., Arribas, S., \& Martinez-Roger, C. 1996, A\&AS, 117, 227
Bagnulo, S., Jehin, E., Ledoux, C., et al. 2003, The Messenger, 114, 10 Bailer-Jones, C. A. L. 2000, A\&A, 357, 197

Barklem, P. S., Christlieb, N., Beers, T. C., et al. 2005, A\&A, 439, 129

Bergemann, M., Lind, K., Collet, R., Magic, Z., \& Asplund, M. 2012, MNRAS, 427, 27

Boeche, C., Siebert, A., Williams, M., et al. 2011, AJ, 142, 193

Bond, H. E., Nelan, E. P., VandenBerg, D. A., Schaefer, G. H., \& Harmer, D. 2013, ApJ, 765, L12

Bonifacio, P. 2005, Mem. Soc. Astron. It. Suppl., 8, 114

Bonifacio, P., \& Caffau, E. 2003, A\&A, 399, 1183

Bonifacio, P., Sbordone, L., Caffau, E., et al. 2012, A\&A, 542, A87

Bouchy, F., \& Sophie Team 2006, in Tenth Anniversary of 51 Peg-b: Status of and prospects for hot Jupiter studies (Paris: Frontier Group), 319

Caffau, E., Bonifacio, P., François, P., et al. 2011a, Nature, 477, 67

Caffau, E., Ludwig, H.-G., Steffen, M., Freytag, B., \& Bonifacio, P. 2011b, Sol. Phys., 268, 255

Caffau, E., Bonifacio, P., François, P., et al. 2012, A\&A, 542, A51

Caffau, E., Bonifacio, P., Sbordone, L., et al. 2013, A\&A, 560, A71

Casagrande, L., Ramírez, I., Meléndez, J., Bessell, M., \& Asplund, M. 2010, A\&A, 512, A54

Castelli, F. 2005, Mem. Soc. Astron. It. Suppl., 8, 25

Chamberlain, J. W., \& Aller, L. H. 1951, ApJ, 114, 52

Cox, A. N. 2000, Allen's Astrophysical Quantities (Springer)

Dekker, H., D’Odorico, S., Kaufer, A., Delabre, B., \& Kotzlowski, H. 2000, Proc. SPIE, 4008, 534

Erspamer, D., \& North, P. 2002, A\&A, 383, 227

François, P., Depagne, E., Hill, V., et al. 2003, A\&A, 403, 1105

Fulbright, J. P. 2000, AJ, 120, 1841

Gilmore, G., Randich, S., Asplund, M., et al. 2012, The Messenger, 147, 25

González Hernández, J. I., \& Bonifacio, P. 2009, A\&A, 497, 497

Gratton, R. G., Sneden, C., Carretta, E., \& Bragaglia, A. 2000, A\&A, 354, 169

Holweger, H., Gehlsen, M., \& Ruland, F. 1978, A\&A, 70, 537

Hosford, A., Ryan, S. G., García Pérez, A. E., Norris, J. E., \& Olive, K. A. 2009, A\&A, 493, 601

Hosford, A., García Pérez, A. E., Collet, R., et al. 2010, A\&A, 511, A47

Katz, D., Soubiran, C., Cayrel, R., Adda, M., \& Cautain, R. 1998, A\&A, 338, 151

Koch, A., \& McWilliam, A. 2008, AJ, 135, 1551

Kurucz, R. L. 2005, Mem. Soc. Astron. It. Suppl., 8, 14

Lodders, K., Plame, H., \& Gail, H.-P. 2009, Landolt-Börnstein - Group VI Astronomy and Astrophysics Numerical Data and Functional Relationships in Science and Technology, Solar System, ed. J. E. Trümper, 4, 44

Meléndez, J., Bergemann, M., Cohen, J. G., et al. 2012, A\&A, 543, A29

Magrini, L., Randich, S., Friel, E., et al. 2013, A\&A, 558, A38

Mucciarelli, A., Pancino, E., Lovisi, L., Ferraro, F. R., \& Lapenna, E. 2013, ApJ, 766, 78

Pasquini, L., Avila, G., Blecha, A., et al. 2002, The Messenger, 110, 1

Perruchot, S., Kohler, D., Bouchy, F., et al. 2008, Proc. SPIE, 7014,

Posbic, H., Katz, D., Caffau, E., et al. 2012, A\&A, 544, A154

Prugniel, P., Vauglin, I., \& Koleva, M. 2011, A\&A, 531, A165

Recio-Blanco, A., Bijaoui, A., \& de Laverny, P. 2006, MNRAS, 370, 141

Sbordone, L. 2005, Mem. Soc. Astron. It. Suppl., 8, 61

Sbordone, L., Bonifacio, P., Castelli, F., \& Kurucz, R. L. 2004, Mem. Soc. Astron. It. Suppl., 5, 93

Sousa, S. G., Santos, N. C., Israelian, G., Mayor, M., \& Monteiro, M. J. P. F. G. 2007, A\&A, 469, 783

Sousa, S. G., Santos, N. C., Israelian, G., Mayor, M., \& Udry, S. 2011, A\&A, 533, A141

Stetson, P. B., \& Pancino, E. 2008, PASP, 120, 1332

Valenti, J. A., \& Piskunov, N. 1996, A\&AS, 118, 595

Van der Swaelmen, M., Hill, V., Primas, F., \& Cole, A. A. 2013, A\&A, 560, A44

Vernet, J., Dekker, H., D’Odorico, S., et al. 2011, A\&A, 536, A105

Wu, Y., Singh, H. P., Prugniel, P., Gupta, R., \& Koleva, M. 2011, A\&A, 525, A71

Yanny, B., Rockosi, C., Newberg, H. J., et al. 2009, AJ, 137, 4377

York, D. G., Adelman, J., Anderson, John, E., Jr., et al. 2000, AJ, 120, 1579 